\title{
Dynamical analysis of blocking events: spatial and temporal fluctuations of covariant Lyapunov vectors
}

Article

Accepted Version

Schubert, S. and Lucarini, V. (2016) Dynamical analysis of blocking events: spatial and temporal fluctuations of covariant Lyapunov vectors. Quarterly Journal of the Royal

Meteorological Society, 142 (698). pp. 2143-2158. ISSN 1477870X doi: https://doi.org/10.1002/qj.2808 Available at https://centaur.reading.ac.uk/71494/

It is advisable to refer to the publisher's version if you intend to cite from the work. See Guidance on citing.

To link to this article DOI: http://dx.doi.org/10.1002/qj.2808

Publisher: Royal Meteorological Society

All outputs in CentAUR are protected by Intellectual Property Rights law, including copyright law. Copyright and IPR is retained by the creators or other copyright holders. Terms and conditions for use of this material are defined in the End User Agreement.

www.reading.ac.uk/centaur 
Central Archive at the University of Reading

Reading's research outputs online 


\title{
Dynamical Analysis of Blocking Events: Spatial and Temporal Fluctuations of Covariant Lyapunov Vectors
}

\author{
Sebastian Schubert ${ }^{1,2}$ and Valerio Lucarini ${ }^{2,3,4}$ \\ ${ }^{1}$ IMPRS - ESM, MPI für Meteorologie, University of Hamburg, Hamburg, Germany, \\ Email: sebastian.schubert@mpimet.mpg.de \\ ${ }^{2}$ Meteorological Institute, CEN, University Of Hamburg, Hamburg, Germany \\ ${ }^{3}$ Department of Mathematics and Statistics, University of Reading, Reading, United \\ Kingdom \\ ${ }^{4}$ Walker Institute for Climate System Research, University of Reading, Reading, \\ United Kingdom
}

First Draft: November 2015

This Draft: January 2016

\begin{abstract}
One of the most relevant weather regimes in the mid-latitudes atmosphere is the persistent deviation from the approximately zonally symmetric jet stream to the emergence of so-called blocking patterns. Such configurations are usually connected to exceptional local stability properties of the flow which come along with an improved local forecast skills during the phenomenon. It is instead extremely hard to predict onset and decay of blockings. Covariant Lyapunov Vectors (CLVs) offer a suitable characterization of the linear stability of a chaotic flow, since they represent the full tangent linear dynamics by a covariant basis which explores linear perturbations at all time scales. Therefore, we assess whether CLVs feature a signature of the blockings. As a first step, we examine the CLVs for a quasi-geostrophic beta-plane two-layer model in a periodic channel baroclinically driven by a meridional temperature gradient $\Delta T$. An orographic forcing enhances the emergence of localized blocked regimes. We detect the blocking events of the channel flow with a Tibaldi-Molteni scheme adapted to the periodic channel. When blocking occurs, the global growth rates of the fastest growing CLVs are significantly higher. Hence, against intuition, the circulation is globally more unstable in blocked phases. Such an increase in the finite time Lyapunov exponents with respect to the long term average is attributed to stronger barotropic and baroclinic conversion in the case of high temperature gradients, while for low values of $\Delta T$, the effect is only due to stronger barotropic instability. In order to determine the localization of the CLVs we compare the meridionally averaged variance of the CLVs during blocked and unblocked phases. We find that on average the variance of the CLVs is clustered around the center of blocking. These results show that the blocked flow affects all time scales and processes described by the CLVs.
\end{abstract}

\section{Introduction}

The study of weather regimes in the atmosphere is a key topic in meteorology and geosciences. In particular, blocking highs have been early on identified as persistent, large scale deviations from the zonally symmetric general circulation Rex, 1950, Baur, 1947. Traditionally, the detection and description of these events employs objective indicators based on pressure anomalies in the atmosphere obtained from observational data or output of general circulation models Lejenäs and $\{\backslash \mathrm{O}\} \mathrm{kland}$, 
1983, Tibaldi and Molteni, 1990, Schalge et al., 2011]. Such blocking events and related large scale weather regimes provide an important contribution to the low frequency variability of the atmosphere. In particular, one can interpret the mid-latitude atmosphere as jumping between a zonal regime and a blocked regime, or, more in general, a regime where long waves are strongly enhanced Benzi et al., 1986, Sutera, 1986, Molteni et al., 1988, Ruti et al., 2006. One needs to remark that the socalled bimodality theory and the analyses which have confirmed - at least partially - its validity have been criticized in the literature, see e.g. Nitsche et al. [1994] and Ambaum 2008. In Charney and DeVore 1979, Charney and Straus 1980, it was speculated that the existence of multiple stationary equilibria in simple models of the atmospheric circulation is the root cause for weather regimes. In their investigation of a highly truncated quasi-geostrophic (QG) models, several stationary states exist due to an orographic forcing. Different weather regimes are then associated with the neighborhood of the various stationary states. Contrary to this theory of multiple equilibria, it was found that in less severely truncated models, which adopted realistic forcings, stationary states are far away from the attractor and/or only one stationary state exists Reinhold and Pierrehumbert, 1982, Tung and Rosenthal, 1985, Speranza and Malguzzi, 1988]. In a recent contribution by Faranda et al. [2015], a different paradigm is instead proposed: blocking events are seen as close returns to an unstable fixed point in a suitably defined reduced space describing the large scale dynamics of the atmosphere.

When considering high-dimensional chaotic dynamics, we have to look at the problem of the possible existence of weather regimes by looking at the properties of the invariant measure supported on the attractor of the system. A possible way to revisit the idea of transitions between atmospheric regimes is based on looking at the switching between the neighborhood of unstable periodic orbits Gritsun 2013. We remind that unstable periodic orbits provide an alternative way to reconstruct the properties of the attractor of a chaotic dynamical systems (Cvitanovic and Eckhard 1991). Also, heteroclinic connections between unstable stationary states were found in a highly truncated barotropic model Crommelin 2003. In models with higher complexity leftovers of these structures are found and correlate with transitions between different weather regimes Kondrashov et al., 2004, Sempf et al. 2007]. In a reduced model phase space, this allows for identifying different dynamically stable weather regimes and less stable transitions paths between them Tantet et al., 2015.

In this paper, we take inspiration from the classical point of view on the dynamics of blocking, which focuses on the analysis of the linear instabilities of low-order models, but here we we consider more Earth-like - at least, qualitatively - background turbulent atmospheric conditions. While the attractors we consider are strange geometrical objects, we follow a mathematical approach such that we are able to stick to the investigation of linear stability properties, which allows for a relatively easy interpretation of the underlying physical mechanisms. Ever since Lorenz [1963], it is clear that linear stability is a measure of predictability of the atmosphere. Therefore, the difficulty of predicting - in time - the onset and decay of weather regimes and their persistence should be reflected in local stability properties. The analysis of optimal linear perturbations indicated that the leading optimal perturbation localizes where blocking occurs Buizza and Molteni, 1996. In a study by Frederiksen [1997 normal modes for a time varying basic state were investigated. Naoe and Matsuda 2002 found that - in contrast to the baroclinic instability - the emergence of blocking events can not be explained by linear perturbations of fixed states of the atmosphere, instead non-linear processes have to be included.

Our approach to this problem will be based on investigating blocking events using Covariant Lyapunov Vectors (CLVs). These vectors form a norm-independent and covariant basis of the tangent linear space Ruelle, 1979, Eckmann and Ruelle, 1985, Trevisan and Pancotti, 1998, Ginelli et al. 2007. The long-time average of the growth rates of the CLVs give the Lyapunov exponents (LEs), see discussion in Froyland et al. 2013 and Vannitsem and Lucarini 2015. Note that by spanning the tangent space of the attractor, CLVs allow in principle a precise calculation of the response operator to an arbitrary perturbation of a dynamical system Lucarini et al., 2014, Lucarini and Sarno, 2011, Ruelle, 2009]. CLVs provide a powerful method for characterizing the properties of weather regimes. First, they are a first order representation of the dynamics around a fully nonlinearly evolving background state, so that no simplifying hypotheses are made on the dynamics. Second, they are a generalization of the normal mode instabilities of basic states of the atmosphere, 
so that it is still possible to use all the machinery of linear ordinary differential equations. Taking these points into consideration, it is suggestive to consider CLVs as a superior choice over other orthogonal, hence norm-dependent Lyapunov vectors [Legras and Vautard, 1996].

Previously, we have investigated CLVs in a quasi geostrophic two layer model in a periodic channel Schubert and Lucarini, 2015. In that work, we addressed how the average energy and momentum transports of the CLVs are related to their growth and decay in respect to the background state and how they explain the variance of the background state. Moreover, we provided a bridge between the growth rate of the CLVs and the physical mechanisms responsible for the variability of the quasigeostrophic flow, namely the barotropic and baroclinic conversion, by a detailed analysis of the Lorenz Energy cycle of each CLV. We note that our focus was exclusively on the long-term properties of the flow, of its CLVs, and of the corresponding LEs.

In this paper, we are concerned with weather regimes in the background state, hence we will study the fluctuations of the CLVs and of the finite-time LEs. The rationale of our study is then the following. Using the classical Tibaldi-Molteni scheme blocking detection, we will determine when the flow is unblocked and when/where the flow switches to a blocked state [Tibaldi and Molteni, 1990]. We will then address two questions.

1. Is there a systematic signature of blocked phases in the growth rates of the linear perturbations? Is there a systematic change in the energetics of the flow?

2. Is the occurrence of blocked phases linked to the presence of specific patterns for the CLVs and to their localization in the physical space?

Note that the second question is different from the average localization of the CLVs investigated in Szendro et al. 2008. In order to address these questions, we have extended the model of our previous study with an orographic forcing, following Charney and Straus, 1980. As in our previous study, the model is baroclinically driven by introducing a relaxation meridional temperatrue gradient $\Delta T$ and dissipates energy via Ekman pumping, which parameterizes the effect of the planetary boundary layer. The orography in our investigation is a Gaussian bump in the middle of the domain with horizonal scale of $O(1000) \mathrm{km}$. We explore the sensitivity of the problem by considering multiple setups featuring different heights of the gaussian bump and different values of $\Delta T$. The various setups all exhibit chaotic conditions with many positive LEs.

We find that blocking increases with a higher meridional temperature gradient $\Delta T$ and is additionally enhanced by orography, which, by breaking the zonal symmetry, contributes as a catalyst to the process of a phase lock mechanism that allows standing perturbations to grow, as envisioned in Benzi et al. [1986]. The spatial variance of the CLVs is dominantly located around the region where blocking occurs when compared to the average variance during unblocked phases. Furthermore, the growth rates of the fastest growing CLVs are higher during blocked phases, pointing at the fact that the system has globally a lower predictability during blocked phases, possibly as a result of the difficulty of predicting when the onset and decay of the blocking events. The observed increased instability suggests also that the local dimension of the attractor is higher during blocking. We explain the changed growth behavior by using a generalization of the Lorenz energy cycle between the CLVs and the background state introduced in Schubert and Lucarini [2015]. We find that for high values of $\Delta T$ the increased instability is dominantly caused by an increased input of energy to the CLVs by baroclinic and barotropic conversions, while for weakly baroclinic flows the intensification of barotropic instability is the only active mechanism. We speculate that these results hint at a possible definition of blocking by taking into account the properties of all CLVs at a particular time.

The structure of the paper can be summarized as follows. In section 2, we describe our experimental set-up, by sketching the formulation of the QG model, the basic properties of CLVs, and the blocking detection algorithm. In section 3 , we present our results on the properties of blocked vs unblocked phases, discussing the properties of the fluctuations of LEs and CLVs and investigating the sensitivity of our results to changes in the forcing and in the orography. Finally, in section 4 we give an outlook and summary and point the reader towards future work on these topics. 


\section{Experimental Setup}

\section{$2.1 \quad$ The Model}

We conduct our investigation with a two layer model of the mid-latitudes featuring the basic large scale baroclinic and barotropic processes of the atmosphere. Previously, we have used the same model without orography to obtain the CLVs in Schubert and Lucarini 2015. The model is a spectral version of the classical model introduced by Phillips [1956] extended by an orographic forcing. This type of model was earlier used to investigate blocking and stationary states by Charney and Straus 1980. The horizontal domain is rectangular $(x, y) \in\left[0, L_{x}\right] \times\left[0, L_{y}\right]$. It is periodic in the $\mathrm{x}$-direction and a no-flux condition is imposed at $y=0, \pi$ (see figure 1b). In the vertical, two layers are resolved (see figure 1). We solve the quasi geostrophic vorticity equation at the pressure levels $p_{2}=750 \mathrm{hPa}$ and $p_{1}=250 h P a$ level and the thermodynamic equation at the pressure level $p_{1.5}=500 h P a$ (see figure 1a). The model is forced by a newtonian cooling towards a zonally symmetric temperature

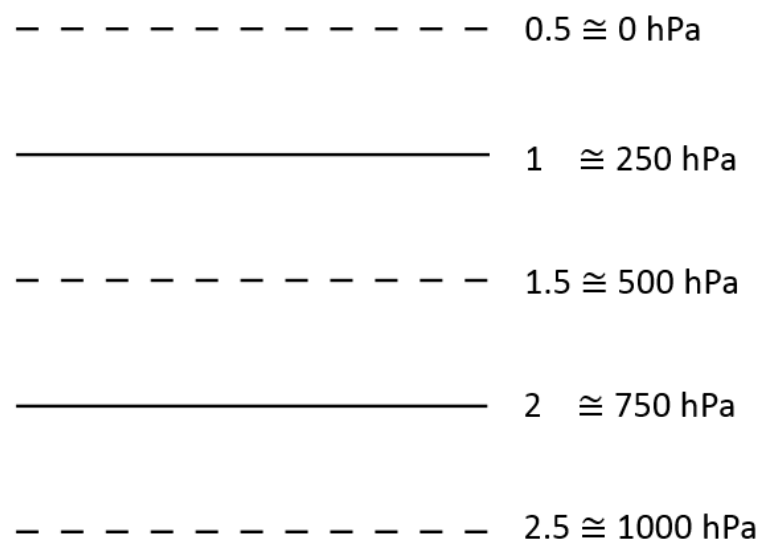

(a) Vertical Structure

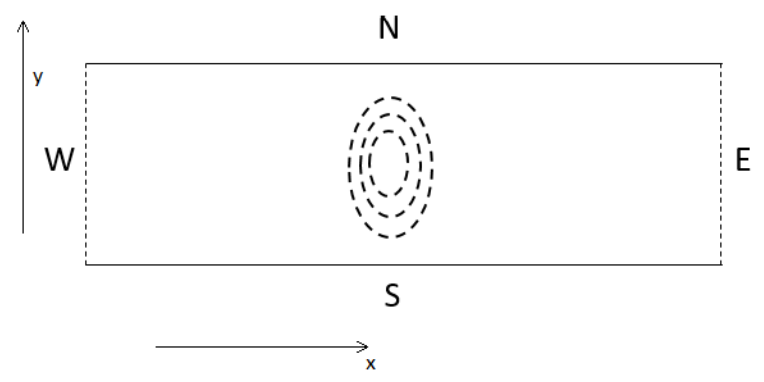

(b) Horizontal Domain

Figure 1: The domain of the QG model. The right panel shows the geometry of the horizontal domain and in dashed lines the position of the orography implemented as a forcing on $\omega_{2.5}$

profile

$$
T_{e}=\frac{\Delta T}{2} \cos \left(\frac{\pi y}{L_{y}}\right)
$$

Small scale interactions are parameterized through eddy diffusion $k_{h} \Delta^{2} \psi$. The interaction with the boundary layer due to Ekman pumping and orography

$$
h_{G B}=h_{0} e^{-\left(\frac{x-L_{x}}{\sigma_{x}}\right)^{2}-\left(\frac{y-L_{y}{ }^{2}}{\sigma_{y}}\right)}
$$

is parameterized by imposing in the lower layer vertical p-velocity $\omega_{2.5}=\frac{\Delta p}{f_{0}} 2 r \Delta \psi_{2}-\frac{\Delta p}{H} J\left(\psi_{2}, h_{G B}\right)$, see, e.g. where $H$ is the height of the atmosphere $(7.3 \mathrm{~km})$. Note that for this implementation of orography we have to ensure that $h / H$ at least smaller than 1 . At the top of the atmosphere $\omega_{0.5}$ is zero. The system is solved in terms of the geostrophic streamfunction $\psi$ and the temperature $T$, see 
Holton 2004.

$$
\begin{aligned}
\frac{\mathrm{d}}{\mathrm{dt}} \Delta \psi_{1}= & -J\left(\psi_{1}, \Delta \psi_{1}+f_{0}+\beta y\right)+f_{0} \frac{\omega_{1.5}-\omega_{0.5}}{\Delta p} \\
& +k_{h} \Delta^{2} \psi_{1} \\
\frac{\mathrm{d}}{\mathrm{dt}} \Delta \psi_{2}= & -J\left(\psi_{2}, \Delta \psi_{2}+f_{0}+\beta y\right)+f_{0} \frac{\omega_{2.5}-\omega_{1.5}}{\Delta p} \\
& +k_{h} \Delta^{2} \psi_{2} \\
\frac{\mathrm{d}}{\mathrm{dt}} T= & -J\left(\psi_{M}, T\right)+S_{p} \omega_{1.5}+r_{R}\left(T_{e}-T\right)+\kappa \Delta T
\end{aligned}
$$

Where equations $1 \mathrm{a}$ and $1 \mathrm{~b}$ are the QG vorticity equation and equation $1 \mathrm{cc}$ is the QG thermodynamic energy equation. For more details on the model itself, we refer to the model description in Schubert and Lucarini 2015]. The adimensionalization is performed according to table 1 . We introduce also a modified stability parameter $S=S_{p} \frac{R \Delta p}{2 f_{0}^{2}}$. The adimensional equations are then the following. We define a barotropic $\psi_{M}=\frac{\psi_{1}+\psi_{2}}{2}$ and baroclinic streamfunction $\psi_{T}=\frac{\psi_{1}-\psi_{2}}{2}$, the latter being proportional to the temperature $T=\frac{2 f_{0}}{R} \psi_{T}$. We then obtain:

$$
\begin{aligned}
\frac{\mathrm{d}}{\mathrm{dt}} \Delta \psi_{M}= & -J\left(\psi_{M}, \Delta \psi_{M}+\beta y\right)-J\left(\psi_{T}, \Delta \psi_{T}\right) \\
& -r \Delta\left(\psi_{M}-\psi_{T}\right)+k_{h} \Delta^{2} \psi_{M} \\
& +\frac{1}{2} J\left(\psi_{M}-\psi_{T}, h_{G B}\right) \\
\frac{\mathrm{d}}{\mathrm{dt}} \Delta \psi_{T}= & -J\left(\psi_{T}, \Delta \psi_{M}+\beta y\right)-J\left(\psi_{M}, \Delta \psi_{T}\right) \\
& +r \Delta\left(\psi_{M}-\psi_{T}\right)+k_{h} \Delta^{2} \psi_{T} \\
& -\frac{1}{2} J\left(\psi_{M}-\psi_{T}, h_{G B}\right)+\omega \\
\frac{\mathrm{d}}{\mathrm{dt}} \psi_{T}= & -J\left(\psi_{M}, \psi_{T}\right)+S \omega+r_{R}\left(\psi_{T e}-\psi_{T}\right) \\
& +\kappa \Delta \psi_{T}
\end{aligned}
$$

The orography $h_{G B}$ is an idealized Gaussian bump designed to resemble loosely the scales of the Rocky Mountains placed in the middle of the horizontal domain. Hence, $\sigma_{x}=1000 \mathrm{~km}$ and $\sigma_{y}=2000 \mathrm{~km}$. We integrate the equations in spectral space using the following decomposition.

$$
\begin{aligned}
& \psi(x, y, t)=\sum_{k, l=1}^{N_{x}, N_{y}}\left(\psi^{r}(k, l, t) \cos (a k x)\right. \\
& \left.+\psi^{i}(k, l, t) \sin (a k x)\right) \sin (l y)+\sum_{l=1}^{N_{y}} \psi^{r}(0, l, t) \cos (l y)
\end{aligned}
$$

The spectral cutoff is in the zonal direction at $N_{x}=10$ and in the meridional direction at $N_{y}=12$. The total dimension of the model phase space is $2 N_{y}\left(2 N_{x}+1\right)=504$. This resolution is rather coarse but nevertheless still sufficient for capturing the large scale structure that we are interested in, see Schubert and Lucarini 2015. We perform a spin up run of 30 years. All results will be based on a time series of 31 years. We investigate three different mountain heights $h_{0}(1.48 \mathrm{~km}, 2.96 \mathrm{~km}$ and $4.44 \mathrm{~km})$ and four different meridional temperature gradients $\Delta T(40 \mathrm{~K}, 50 \mathrm{~K}, 66 \mathrm{~K}$ and $76 \mathrm{~K})$. This ensures the investigation of different states of large scale turbulence and the assessment of the impact of orography. In control runs, the experiments are done without orography. The implemented $4^{\text {th }}$ order Runge-Kutta-Scheme uses a fixed time step of 2.77 hours ( 1 adimensional time unit) except for 


\begin{tabular}{|c|c|c|c|c|c|}
\hline $\begin{array}{l}\text { Variables, Operators } \\
\text { \& Constants }\end{array}$ & Symbol & Unit & $\begin{array}{l}\text { Scaling } \\
\text { Factor }\end{array}$ & $\begin{array}{l}\text { Value of } \\
\text { Scaling Factor }\end{array}$ & \\
\hline Streamfunction & $\psi$ & $m^{2} / s$ & $L^{2} f_{0}$ & $10^{10} / \pi^{2}$ & \\
\hline Temperature & $T$ & $K$ & $2 f_{0}^{2} L^{2} / R$ & 705.97 & \\
\hline Velocity & $\mathbf{v}$ & $\mathrm{m} / \mathrm{s}$ & $L f_{0}$ & $10^{3} / \pi$ & \\
\hline Laplace Operator & $\Delta$ & $1 / m^{2}$ & $1 / L^{2}$ & $\pi^{2} / 10^{14}$ & \\
\hline Vertical p-Velocity & $\bar{\omega}$ & $\mathrm{Pa} / \mathrm{s}$ & $\Delta p f_{0}$ & 0.01 & \\
\hline Jacobian & $J(\cdot, \cdot)$ & $1 / m^{2}$ & $1 / L^{2}$ & $\pi^{2} / 10^{14}$ & \\
\hline Parameters & Symbol & $\begin{array}{l}\text { Dimensional } \\
\text { Value }\end{array}$ & Unit & $\begin{array}{l}\text { Scaling } \\
\text { Factor }\end{array}$ & $\begin{array}{l}\text { Non-dimensonal } \\
\text { Value }\end{array}$ \\
\hline $\begin{array}{l}\text { Forced Meridional } \\
\text { Temperature Gradient }\end{array}$ & $\Delta T$ & $40-76$ & $K$ & $2 f_{0}^{2} L^{2} / R$ & $0.0567-0.1076$ \\
\hline Height Of Mountain & $h_{0}$ & $1.48,2.96,4.44$ & $\mathrm{~km}$ & $H$ & $0.2,0.4,0.6$ \\
\hline Width of Mountain & $\sigma_{x}, \sigma_{y}$ & 1000,2000 & $k m$ & $L$ & $0.1 \pi, 0.2 \pi$ \\
\hline Eddy-Heat Diffusivity & $\kappa$ & $10^{5}$ & $m^{2} / s$ & $L^{2} f_{0}$ & $9.8696 \cdot 10^{-5}$ \\
\hline Eddy-Momentum Diffusivity & $k_{h}$ & $10^{5}$ & $m^{2} / s$ & $L^{2} f_{0}$ & $9.8696 \cdot 10^{-5}$ \\
\hline Thermal Damping & $r_{R}$ & $1.157 \cdot 10^{-6}$ & $1 / s$ & $f_{0}$ & 0.011 \\
\hline Ekman Friction & $r$ & $2.2016 \cdot 10^{-6}$ & $1 / s$ & $f_{0}$ & 0.022 \\
\hline Stability Parameter & $S$ & $3.33 \cdot 10^{11}$ & $m^{2}$ & $L^{2}$ & 0.0329 \\
\hline Coriolis Parameter & $f_{0}$ & $10^{-4}$ & $1 / s$ & $f_{0}$ & 1 \\
\hline Beta & $\beta$ & $1.599 \cdot 10^{-11}$ & $1 /(m s)$ & $f_{0} / L$ & 0.509 \\
\hline Aspect Ratio & $a$ & 0.6896 & 1 & - & 0.6896 \\
\hline Zonal Length & $L_{x}$ & $2.9 \cdot 10^{7}$ & $m$ & $\mathrm{~L}$ & $\frac{2 \pi}{a}$ \\
\hline Meridional Length & $L_{y}^{\infty}$ & $10^{7}$ & $m$ & $\mathrm{~L}$ & $\pi^{a}$ \\
\hline Height Of Atmosphere & $H$ & 7.4 & $k m$ & & \\
\hline Specific Gas Constant & $R$ & 287.06 & $J /(k g K)$ & $R / 2$ & 2 \\
\hline Pressure & $\Delta p$ & $500 h P a$ & $N / m^{2}$ & $\Delta p$ & 1 \\
\hline
\end{tabular}

Table 1: Parameters and Variables used in this model and the respective adimensionalization scheme. Note that the scales for time and length are $t=10^{4} s=1 / f_{0}$ and $L=\frac{10^{7}}{\pi} m$ 
the highest $\Delta T=76 \mathrm{~K}$, where, instead, we choose a time step of 1.385 hours ( 0.5 adimensional time units). The analysis of the data is sampled every 2.77 hours. For our analysis, we investigate a total time series of 31 years. For this we consider additionally 15 years as spin up before and after the 31 years in order to compute the CLVs.

\subsection{Covariant Lyapunov Vectors}

CLVs are a powerful tool for investigating the tangent linear model of a dynamical system. We have previously summarized theory of CLVs in Schubert and Lucarini 2015 and how they can be obtained via the algorithm of Ginelli et al. [2007].

Let us briefly report on the main properties of the CLVs and their importance for characterizing the dynamics of small perturbations. Summarizing, they represent the covariant directions of expansion and decay in the linear tangent space which grow on average with the LEs, see Legras and Vautard 1996. If the background state is a simple fixed point, they reduce to the classical normal modes of stationary solutions, see Wolfe and Samelson 2007]. For periodic background, they coincide with the Floquet vectors [Floquet, 1883, Samelson, 2001, Wolfe and Samelson, 2006, 2008. These properties make them an ideal choice to describe the growth (in linear approximation) of actual physical disturbances, since the classical Lyapunov vectors (Gram-Schmidt vectors) are orthogonal and can therefore not describe the covariant evolution of nearby trajectories of the background flow, see Pazó et al. [2010, Kuptsov and Parlitz 2012. Following Szendro et al. 2008], we also expect that in the specific case the system is prepared in such a way that a localized perturbation breaks otherwise symmetric boundary conditions, one can observe localization phenomena (not exclusively in the vicinity of the perturbation) for the CLVs of the system. This last property is extremely attractive for the problem studied in this paper.

The evolution of CLVs describes how infinitesimal perturbations added to the background flow $\mathbf{x}_{B}$ change in time. From a dynamical system point of view equation (2) can be written in the following form.

$$
\frac{\mathrm{d}}{\mathrm{dt}} \mathbf{x}=f(\mathbf{x})
$$

An infinitesimal perturbation $\mathbf{v}$ added to $\mathbf{x}_{B}$ solves the following equation which is obtained by conducting a first order expansion of equation (4).

$$
\frac{\mathrm{d}}{\mathrm{dt}} \mathbf{v}_{j}(t)=\sum_{i} \frac{\partial f_{j}}{\partial x_{i}}\left(\mathbf{x}_{B}(t)\right) \mathbf{v}_{i}(t)=: \sum_{i} \mathcal{J}_{j i}\left(\mathbf{x}_{B}(t)\right) \mathbf{v}_{i}(t) .
$$

A normalized, covariant basis for the solutions of this equation is the $\operatorname{CLVs}\left\{\mathbf{c}\left(x_{B}(t)\right)_{j}\right\}_{j=1 . . n}$ (n is here 504). Let us explain what this means more precisely. For this, we need besides the normalized vectors $\mathbf{c}_{j}(t)$ also the corresponding time series of growth rates $\lambda_{i}(t)$. Their average is equal to the jth $\operatorname{LE} \lambda_{j}=\lim _{T \rightarrow \infty} \frac{1}{T} \int_{0}^{T} d t \lambda_{j}(t)$. If we pick a solution $\mathbf{v}$ of equation 5 at time $t$ with the initial condition $\mathbf{v}\left(t_{0}\right)=\mathbf{c}_{j}\left(t_{0}\right)$ then $\mathbf{v}(t)$ has the following form.

$$
\mathbf{v}(t)=e^{\int_{t_{0}}^{t} d t^{\prime} \lambda_{j}\left(t^{\prime}\right)} \mathbf{c}_{j}(t)
$$

Note that the normalized CLVs $\mathbf{c}_{j}(t)$ solve the following slightly altered equation.

$$
\dot{\mathbf{c}}_{j}(t)=\mathcal{J}\left(\mathbf{x}_{B}(t)\right) \mathbf{c}_{j}(t)-\lambda_{j}(t) \mathbf{c}_{j}(t)
$$

Imagine now, we chose an arbitrary initial condition $x_{0}$ at time $t_{0}$, hence a superposition of possibly all CLVs

$$
\mathbf{x}_{0}=\sum_{j} \mathbf{c}_{j}\left(t_{0}\right) A_{j} .
$$

Consequently, the solution $\mathbf{x}$ for equation (5) with $\mathbf{x}(0)=\mathbf{x}_{0}$ has the following form in the basis of the CLVs.

$$
\mathbf{x}(t)=\sum_{j} e^{\int_{t_{0}}^{t} d t^{\prime} \lambda_{j}\left(t^{\prime}\right)} \mathbf{c}_{j}(t) A_{j}
$$


This means the expansion into the CLVs allows it to investigate also very slow growing linear perturbations without interference of the fast growing directions. Note that this is particularly interesting for applications in data assimilation, where models often feature multi scale interactions Pazó et al. 2010]. These properties are unique to CLVs. Other Lyapunov vectors, e.g. Gram-Schmidt vectors, see Kuptsov and Parlitz 2012, are not solutions of equation (5). Hence, CLVs describe to first order solutions of equation (4) that are nearby to $\mathbf{x}_{B}$. Consequently, they have a straightforward physical interpretation and we can obtain meaningful transports and feedbacks of the CLVs connected to the background $\mathbf{x}_{B}$ Schubert and Lucarini, 2015.

For further details on the algorithm to obtain CLVs we refer to Ginelli et al. [2007], Kuptsov and Parlitz 2012 and our previously mentioned previous description in Schubert and Lucarini [2015].

\subsection{Blocking Detection}

We describe briefly the adapted Tibaldi-Molteni scheme Tibaldi and Molteni, 1990 for detecting blocking highs in our model. Since the model is spectral, we are using a Fast-Fourier-Transformalgorithm to transform the spectral fields to a $[64 \times 32]$ grid $(64$ grid points in the $\mathrm{x}$ direction, 32 grid points in the y direction). We will consider only blocking in the barotropic streamfunction $\psi_{P}$, since it is the best representation of the $500 \mathrm{hPa}$ layer in our model discretization. In order to detect blocking high anomalies, we study the occurrence at some longitude of reversals in the direction of the zonal wind with respect to normal conditions. We construct the average zonal wind in the northern and southern sector by constructing the quantities $u_{N}(x, \Delta)=-\delta \psi_{N}(x, \Delta) /\left(y_{N}-y_{0}\right)$ and $u_{S}(x, \Delta)=-\delta \psi_{S}(x, \Delta) /\left(y_{0}-y_{S}\right)$. where $\delta \psi_{N}(x, \Delta)=\psi_{P}\left(x, y_{N}+\Delta\right)-\psi_{P}\left(x, y_{0}+\Delta\right)$ and $\delta \psi_{S}(x, \Delta)=\psi_{P}\left(x, y_{0}+\Delta\right)-\psi_{P}\left(x, y_{S}+\Delta\right)$.

A blocking event occurs, if at a particular coordinate $\mathrm{x} u_{S}$ is negative and $u_{N}$ is sufficiently positive and lasts at least two days. We also allow for a deviation $\Delta$ from the chosen y-coordinates. Summarizing this we have the following criteria.

$$
\begin{aligned}
& u_{N}\left(x, y_{0}+\Delta\right)>9 \mathrm{~m} / \mathrm{s} \\
& u_{S}\left(x, y_{0}+\Delta\right)<0 \mathrm{~m} / \mathrm{s} \\
& y_{N}=8437 \mathrm{~km} ; y_{0}=6250 \mathrm{~km} ; y_{S}=4375 \mathrm{~km} \\
& \Delta=(-940 \mathrm{~km} 0 \mathrm{~km} 940 \mathrm{~km})
\end{aligned}
$$

A word of caution is needed at this point, since the Tibaldi-Molteni index was originally developed for a spherical geometry and considered either observational data or more realistic data taken from GCMs. We still think the use of this index is meaningful, because of its straightforward interpretation and because it describes the presence of an non-zonal deviation from the usually fluctuating, but zonally symmetric jet stream. We will show that the detected blocking events are indeed meaningful, hence a blocked and an unblocked weather regime can be determined (see the following discussion in section 3.1).

\section{Results}

\subsection{Blocking Events}

Let us first look at the blocking rate. Comparing the different setups with the control runs (without orography), we can also assess the impact of the orography on the blocking. For reasons of symmetry, in absence of orography, the statistics of blocking does not depend on $\mathrm{x}$.

The blocking rate (see figure 2 kicks off when $\Delta T$ is larger than $50 \mathrm{~K}$ (even without orography). The orographic forcing creates two to three local maxima in the blocking rate downstream of the peak of orography. These maxima intensify for higher $h_{0}$ and for higher $\Delta T$, but within the range of values considered here the impact of $h_{0}$ reduces for higher $\Delta T$.

As mentioned before, two main configurations of the flow are identified. This can be further substantiated by considering the mean states of the unblocked and blocked flow (e.g. $\Delta T=66 K$ 

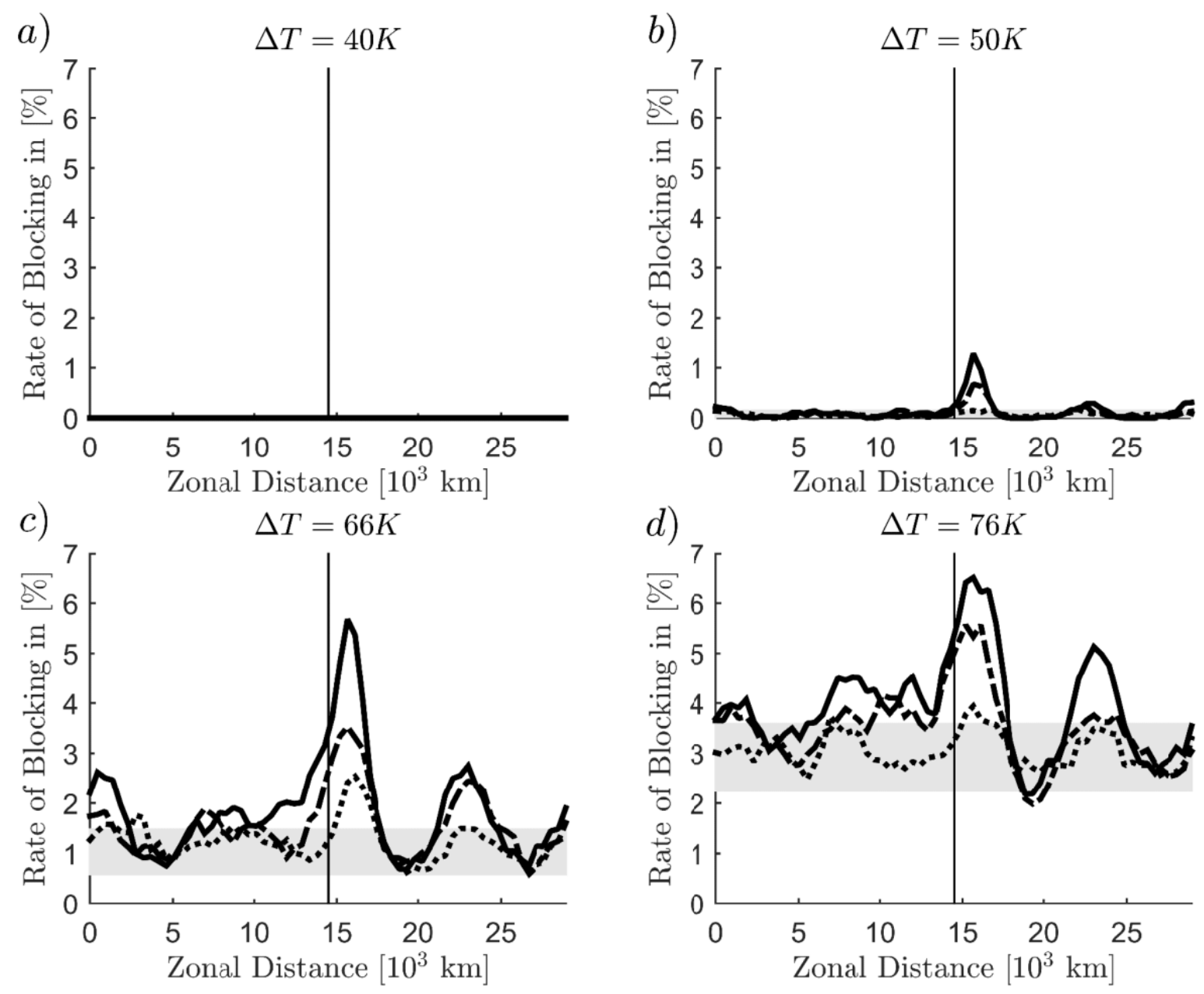

Figure 2: The number of blocked days is the highest behind the peak of the orography. The vertical black line indicates the peak of the orography. The panels show the different values of $\Delta T$, the different heights $h_{0}$ are indicated by the dotted line $(1.48 \mathrm{~km})$, the dash-dotted line $(2.96 \mathrm{~km})$ and the solid line $(4.44 \mathrm{~km})$. Downstream two secondary maxima can be identified. The $\mathrm{x}$ axis indicates the $\mathrm{x}$ coordinate where we detect blocking. The y axis shows in percent the frequency of blocking. The grey shaded area shows the range of the blocking rate along the $\mathrm{x}$ direction without orography.

and $h_{0}=4.44 \mathrm{~km}$ in figure 31. In this way, we treat the unblocked and blocked phases as separate weather regimes and determine the "climate" of the two, respectively. The blocked regime can be further divided into "sub regimes" by considering the mean state over the blocked phase and filtering for those parts of the blocked phase where only a chosen x coordinate is blocked. Since the days where blocking is present are relatively rare, the mean state is computed over the complete time series is more or less identical to the mean state computed, taken over the days where no blocking is observed. Close to the blocked area a clear deviation from the zonal symmetric jet of the mean flow can be seen (the blocking high). We also see that the observed blocking is local and the regions far away from the blocked coordinate do not show large variations with respect to the average unblocked conditions. A first look at the mean unblocked flow shows that it is more zonally symmetric than the mean blocked flow. Nevertheless, there is a non zonal disturbance with wave number four. This is caused by the presence of topographic Rossby waves induced by the orography Holton, 2004. Note that the breaking of Rossby waves is intimately connected to the emergence of blocking events and the meandering of the jet fits roughly to the maxima of the blocking rate Berrisford et al., 2007. The 

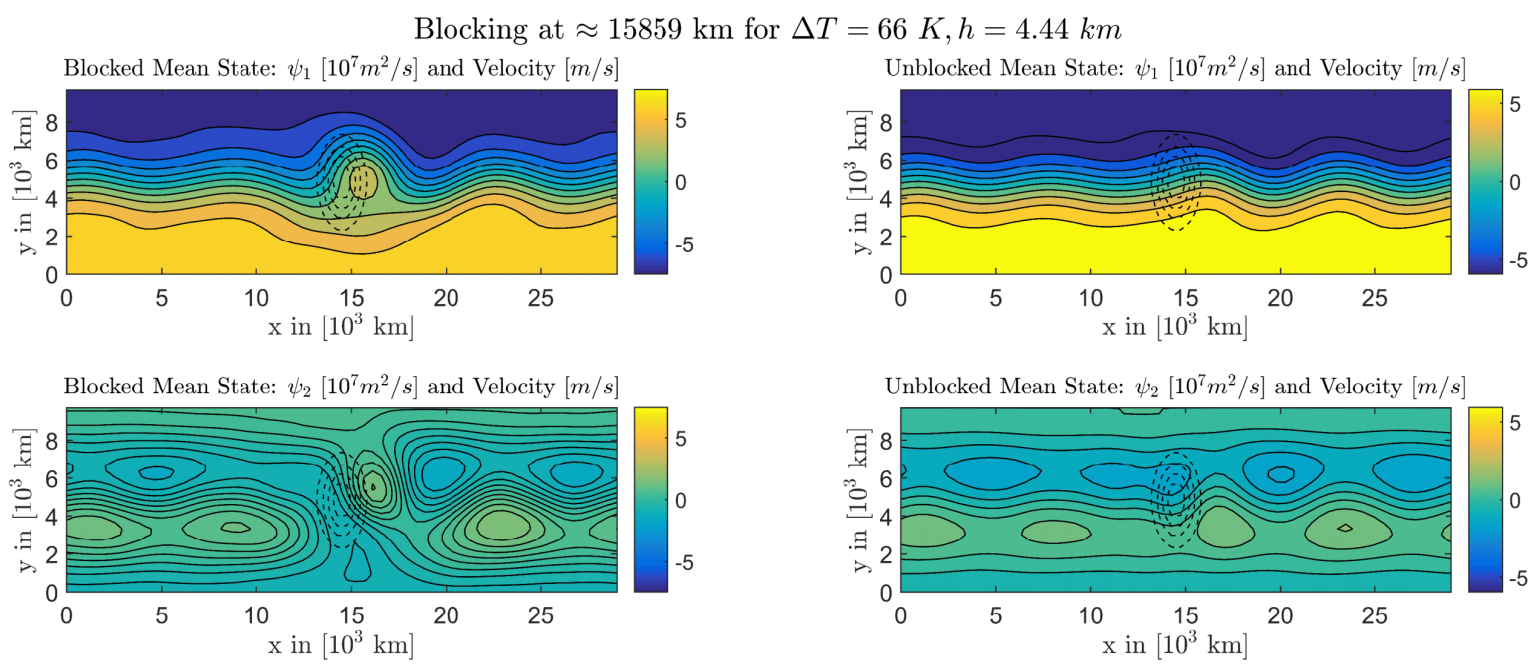

Figure 3: As an example of the observed blocking events, the mean blocked state at $x=15859 \mathrm{~km}$ and the unblocked state for $\Delta T=66 \mathrm{~K}$ and $h=4.44 \mathrm{~km}$ is shown. The left panels show the averaged streamfunction during blocking. Note that, the streamfunction in our QG model should be compared to the stratified streamfunction in models without the hydrostatic balance. This means the upper layer streamfunction $\psi_{1}$ and the lower streamfunction $\psi_{2}$ determine the geostrophic velocities $\mathbf{v}_{1 / 2}=\left(u_{1 / 2}, v_{1 / 2}\right)=\left(-\partial_{y} \psi_{1 / 2}, \partial_{x} \psi_{1 / 2}\right)$. The ageostrophic velocity can then be obtained via the QG momentum equations. The right panels show the average streamfunction for unblocked periods. The upper panels show the upper layer, the lower panels show the lower layer. The dashed lines show the position of orography. The blocking is affecting the flow only locally, since away from the blocking the flow is the mean unblocked flow. We get similar results for blockings at different $\mathrm{x}$ coordinates and different values of $\Delta T$ and $h_{0}$.

results shown in figure 3 do not change significantly for the other setups and other locations, besides the shift of the blocking high to the corresponding $\mathrm{x}$ coordinate.

Let us turn our attention towards the number of blockings and their duration. We show the results for the position of the maximum of the blocking rate (see figure 4), but the findings are similar at other $\mathrm{x}$ coordinates. The average blocking length (lifetime) is only marginally changed, whereas the total number of blockings is increased significantly by orography.

For blocking rate and length (see figures 2 and 4), it appears that as discussed above, adding orography creates preferential geographical locations for the occurrence of blocking. Looking at the global statistics, we have that, for a given value of $\Delta T$, the number of blocking events and the number of blocked days increase with $h_{0}$, even if the catalyzing effect of orographic disturbances is relatively weaker when $\Delta T$ is large enough. The effect of $\Delta T$ on the blocking rate and the number of blockings is also found in observations since the meridional temperature gradient is higher in winter and is associated with a higher blocking rate [Tibaldi and Molteni, 1990. Since we are using an extremely simple model of the atmosphere, it is also not surprising, that the blocking rates and lifetimes do not match quantitatively with observations. The lack of many dynamical and physical ingredients in our model is likely to be responsible for the fact that life times of blocking events are, to a good approximation, exponentially distributed, as opposed to the heavy tail properties found in Pelly and Hoskins 2003. They also define so called sector blocking which detects blocking which have a considerable size in the zonal direction. Given the rather coarse resolution of our model the observed blockings have at least an extent of roughly $1500 \mathrm{~km}$ which means that they already extent over a large area. Moreover, a discrimination of the events according to the blocking lengths (e.g. 2 - 3 days, 3 - 4 days, 4 - 5 days and 5 - 6 days) does not show any significant differences in the observed blocking rates.

We conclude that despite the mentioned limitations the blocking index allows a meaningful def- 

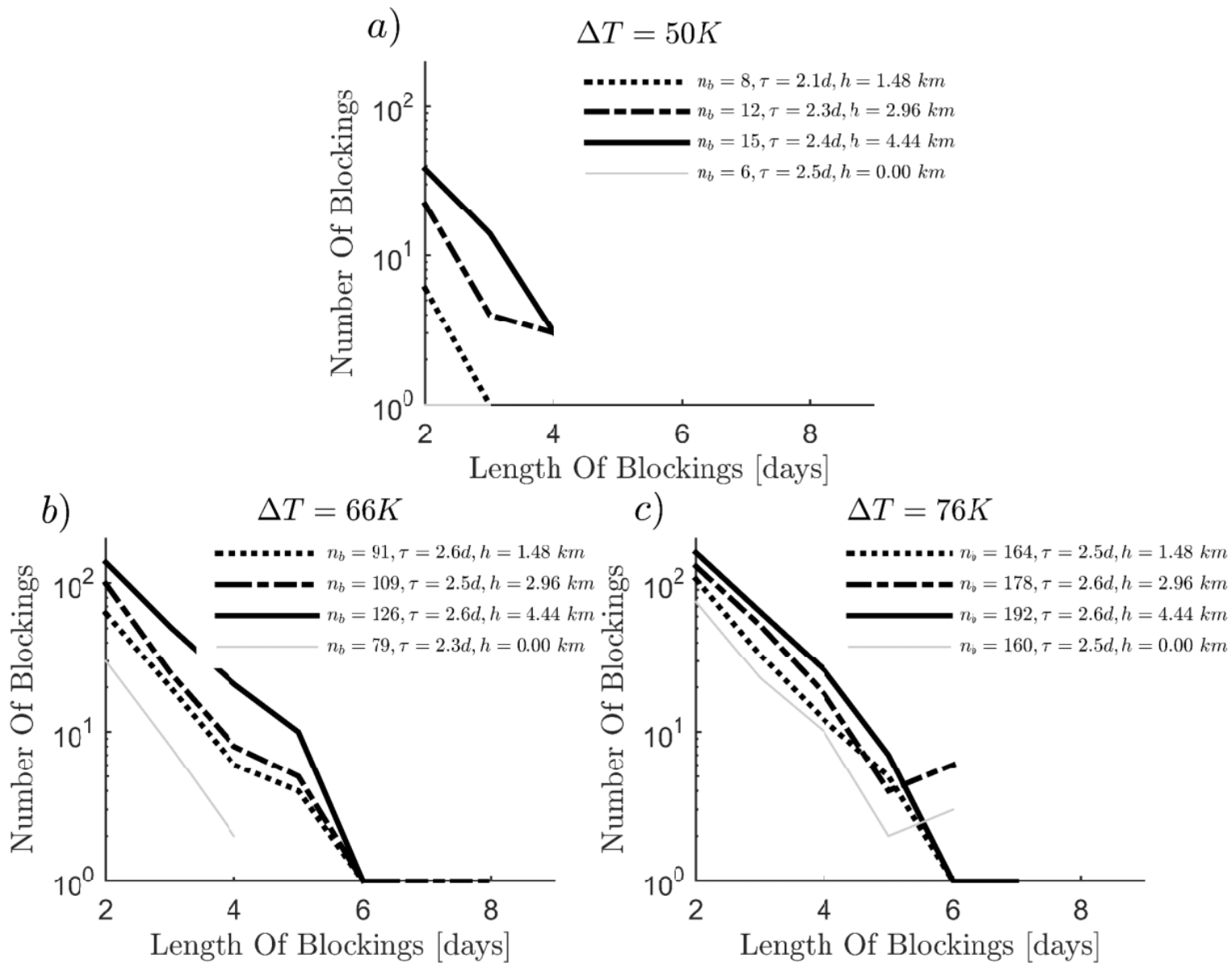

Figure 4: The distribution of blocking lengths at $x=15859 \mathrm{~km}$ with orography in comparison to the control run without orography (light grey). The legends show also the lifetime $\tau$ of the blocking events and the number of blocked days per year $n_{b}$. The y-axis has a log scaling. The total length of the time series is 115705 days (31.7 years).

inition of a blocked and an unblocked regime and that our model responds in at least qualitatively correct way to changes in the orography and meridional temperature gradient.

\subsection{Linear Stability Of Blocking States}

After having clarified in section 3.1 that we indeed observe blocking events induced by orography, we will now evaluate the characteristics of the CLVs during blocked and unblocked phases. We follow up from the previous section and use the distinction between occurrence of blocking events and regular conditions to partition the attractor of the system, and then compute separately the statistical properties of CLVs and finite-time LEs in the two regions.

Let us start by examining basic dynamical and geometrical properties of the system. Starting from the LEs we can derive the Kaplan-Yorke dimension and the metric entropy, see tables 2 and 3 . We assume, as often implicitly done, that the chaotic hypothesis Gallavotti and Cohen 1995 holds. Therefore, we assume that our system has Axiom A-like properties and, in particular, that one has a Sinai-Ruelle-Bowen measure supported on its attractor and describing its asymptotic statistical and dynamical properties. The Kaplan-Yorke dimension is defined as $D_{K Y}=k+\frac{\sum_{i=1}^{k} \lambda_{i}}{\left|\lambda_{k+1}\right|}$, where $k$ is chosen so that the sum of the first $k$ LEs is positive and the sum of the first $k+1$ is negative. This 
Table 2: Properties of the attractor without Orography

\begin{tabular}{ccccc}
\hline$\Delta T[\mathrm{~K}]$ & $\begin{array}{c}\text { Positive } \\
\text { Exponents }\end{array}$ & $\begin{array}{c}\text { Kaplan-Yorke } \\
\text { Dimension }\end{array}$ & $\begin{array}{c}\text { Metric } \\
\text { Entropy }[1 / \text { day }]\end{array}$ & $\begin{array}{c}1 / \lambda_{1} \\
{[\text { day }]}\end{array}$ \\
\hline 39.81 & 17 & 35.83 & 0.25 & 28.8 \\
49.77 & 55 & 125.82 & 3.15 & 6.8 \\
66.36 & 88 & 206.80 & 12.51 & 2.6 \\
76.31 & 98 & 232.09 & 18.66 & 1.9 \\
\hline
\end{tabular}

Table 3: Properties of the attractor with Orography

\begin{tabular}{cccccc}
\hline$\Delta T[\mathrm{~K}]$ & Height $[\mathrm{km}]$ & $\begin{array}{c}\text { Positive } \\
\text { Exponents }\end{array}$ & $\begin{array}{c}\text { Kaplan-Yorke } \\
\text { Dimension }\end{array}$ & $\begin{array}{c}\text { Metric } \\
\text { Entropy }[1 / \text { day }]\end{array}$ & $\begin{array}{c}1 / \lambda_{1} \\
{[\text { day }]}\end{array}$ \\
\hline \multirow{3}{*}{39.81} & 1.48 & 18 & 38.11 & 0.29 & 26.8 \\
& 2.96 & 19 & 41.51 & 0.35 & 24.5 \\
& 4.44 & 19 & 41.24 & 0.34 & 24.5 \\
\hline \multirow{3}{*}{49.77} & 1.48 & 55 & 126.9 & 3.23 & 6.6 \\
& 2.96 & 55 & 129.6 & 3.42 & 6.4 \\
& 4.44 & 56 & 131.6 & 3.56 & 6.2 \\
\hline \multirow{3}{*}{66.36} & 1.48 & 88 & 207.1 & 12.61 & 2.55 \\
& 2.96 & 88 & 207.5 & 12.72 & 2.54 \\
& 4.44 & 89 & 207.9 & 12.85 & 2.51 \\
\hline \multirow{3}{*}{76.31} & 1.48 & 98 & 232.2 & 18.76 & 1.89 \\
& 2.96 & 99 & 232.2 & 18.82 & 1.89 \\
& 4.44 & 99 & 232.4 & 18.88 & 1.88 \\
\hline
\end{tabular}

Table 4: Metric Entropy during the blocked and the unblocked phase. This is the sum of the in the long term averaged positive LEs of the Backward Lyapunov vectors averaged during the blocked and unblocked phases, respectively.

\begin{tabular}{ccccc}
\hline$\Delta T[\mathrm{~K}]$ & Height $[\mathrm{km}]$ & $\begin{array}{c}\text { Metric Entropy } \\
\text { during blocking }[1 / \text { day }]\end{array}$ & $\begin{array}{c}\text { Metric Entropy } \\
\text { no blocking }[1 / \text { day }]\end{array}$ & $\begin{array}{c}\text { Difference } \\
{[1 / \text { day }]}\end{array}$ \\
\hline \multirow{3}{*}{39.81} & 1.48 & - & 0.29 & - \\
& 2.96 & - & 0.34 & - \\
& 4.44 & - & 0.34 & - \\
\hline \multirow{3}{*}{49.77} & 1.48 & 3.41 & 3.21 & 0.20 \\
& 2.96 & 3.60 & 3.41 & 0.19 \\
& 4.44 & 3.76 & 3.53 & 0.23 \\
\hline \multirow{3}{*}{66.36} & 1.48 & 12.71 & 12.54 & 0.17 \\
& 2.96 & 12.82 & 12.70 & 0.12 \\
& 4.44 & 12.95 & 12.77 & 0.18 \\
\hline \multirow{3}{*}{76.31} & 1.48 & 18.87 & 18.67 & 0.20 \\
& 2.96 & 18.89 & 18.74 & 0.15 \\
& 4.44 & 18.97 & 18.78 & 0.19 \\
\hline
\end{tabular}

dimension is an upper bound of the fractal dimension of the attractor of the system. The metric entropy is given by the sum of the positive LEs and a measure for the information creation Eckmann and Ruelle, 1985. With increasing $\Delta T$ the Kaplan Yorke dimension and the metric entropy grow 
monotonically Lucarini et al. 2007. While the observed motions are indeed chaotic for the studied values of $\Delta T$ and $h_{0}$, we can clearly see from these dynamical indicators that turbulence is much better developed for higher values of $\Delta T$. The impact of the orography on these numbers is small but it shows a small upward trend for larger $h_{0}$. This property shows that predicting weather becomes more complicated, if orography is added, since the characteristic predictability time decreases. We take the inverse of the leading LE $\lambda_{1}$ (in tables 2 and 3 ) as a rough measure for predictability, since the rapid divergence of nearby trajectories is a necessary ingredient for having a fast error growth. Note that different dynamical indicators are better suited to study the actual predictability of a system. A better evaluation of the time scales of the system and the associated predictability could be obtained by studying systematically finite time/finite size LEs and the related multifractal properties Boffetta et al., 1998, 2003, which is outside the scope of this paper.
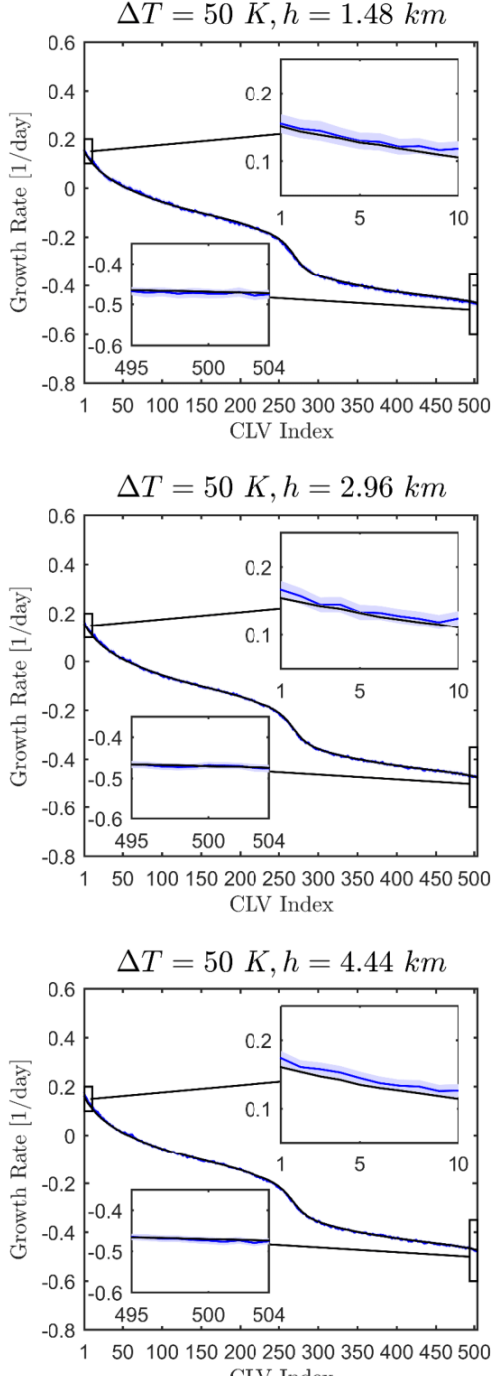

CLV Index
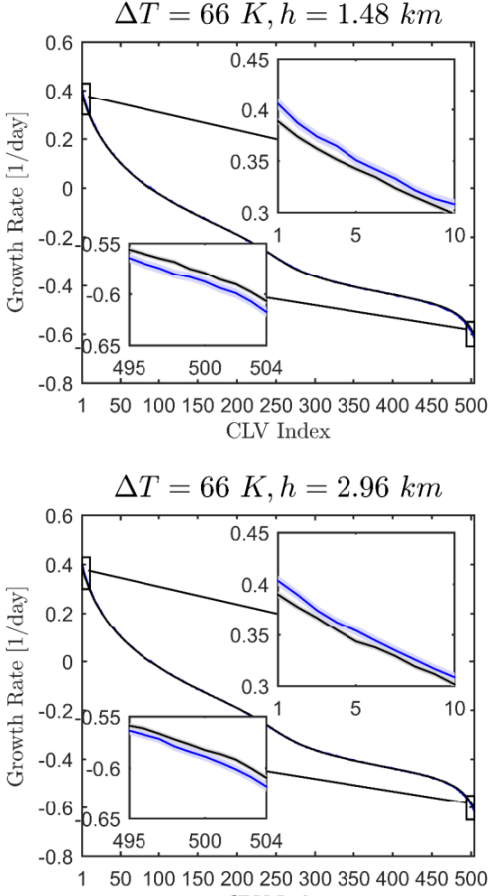

CLV Index

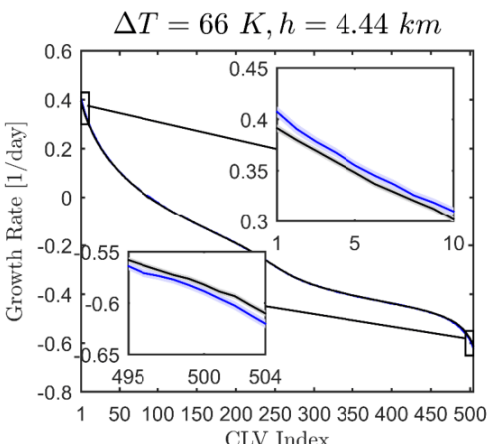

CLV Index
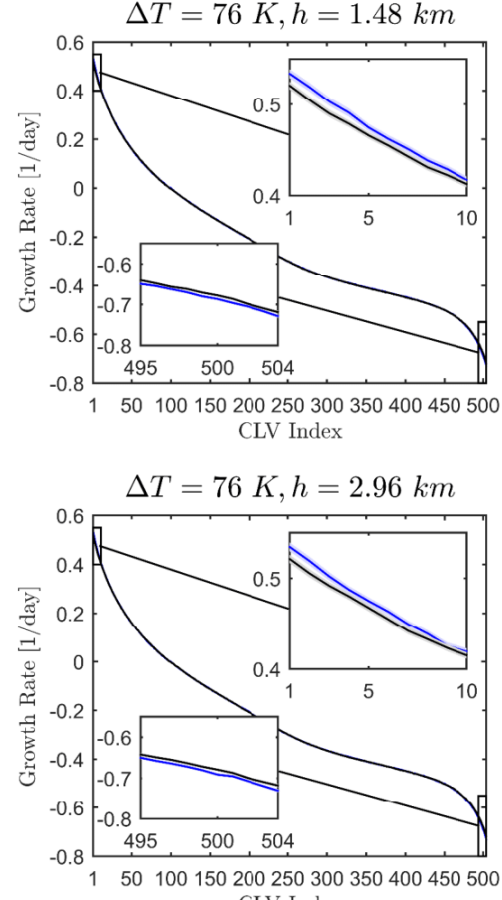

CLV Index

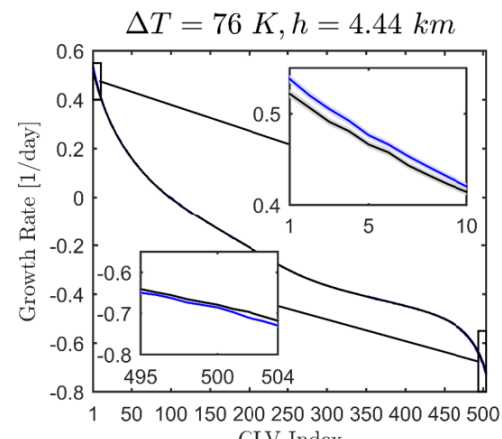

CLV Index

Figure 5: For the nine setups where we observe blocking (see figure 2), the figure shows the differences in the growth rates during blocking (in blue color) versus unblocked phases (in black color). We additionally show the $3 \sigma$ bars of confidence estimated by computing the degrees of freedom of the time series (shaded areas). For $\Delta T=66 K, 76 K$ we can clearly estimate that the CLVs with highest/lowest LEs the baroclinic conversion increases/decreases significantly. For $\Delta T=50 \mathrm{~K}$ such a tendency can not be clearly verified. 
Let us now turn the focus on the average growth rates during the blocked phases and the unblocked phases. Since blocking conditions are relatively rare, at all practical levels this corresponds to comparing the finite time LEs computed during the blocked phase to the actual long-term LEs. The growth rates of the ten leading CLVs increase significantly during blocking for the two largest $\Delta T$. The ten fastest decaying CLVs have significantly higher decay rates during the blocked phase. This result also holds if only blockings with a certain length are considered (e.g. $2-3$ days, $3-4$ days, $4-5$ days and $5-6$ days). The statistical significance is determined by considering the $3 \sigma$ confidence interval which is obtained by computing the degrees of freedom for each time series of the unblocked and blocked growth rates. The degrees of freedom is the number of effective observations of the averages over a fixed width which are not correlated with each other. This width is determined by computing the e-folding time of the autocorrelation of the time series, see Leith 1973 and Mudelsee 2010. This indicates that the unstable CLVs grow globally faster. The question remains whether this is due to changes in the CLVs near or far away from the blocked region. This question will be partly answered in section 3.4 where we will be looking at spatial patterns.

The presence of larger positive LEs during the blocked phases indicate lower predictability. This seems in contradiction with the common knowledge that it is easier to predict the weather during blocking events. One can reconcile these two facts by considering that i) what we consider here is a global measure of predictability, not strictly relate to forecast skill near the region of the blocking; and ii) while predictability is higher during blocking events, it is extremely difficult to predict the onset and the decay of the blocking. Possibly, our result is related to the difficulty of capturing the regime transition. Let us refine a bit our mathematical statements. It is important to recall that predictability is usually characterized by the evolution of phase space volumes, hence the angles between the CLVs have to be considered as well. It is not necessary to actually use the CLVs for answering these questions, since the long term evolution of phase space volumes in the tangent linear regime is given by the the Backward Lyapunov Vectors (also called Gram-Schmidt vectors, see Kuptsov and Parlitz 2012, Schubert and Lucarini 2015]) which grow on average with the LEs, but their finite size LEs are different from the CLVs. In order to characterize the growth of a volume which covers all unstable directions, we use the fluctuations of the BLV-LEs (LEs of the Backward Lyapunov Vectors) to compute the metric entropy which is the sum of all positive LEs [Eckmann and Ruelle, 1985]. In order to discriminate between the blocked and unblocked phase, we then average separately over these two phases of the flow (see table 4). The metric entropy confirms the slightly increased instability observed in the finite time LEs of the CLVs during blocking. We wish to remark that while considering larger values of $h_{0}$ leads to more frequent blocking events, no significant effect is instead found on the average growth rate of the disturbances. Orography plays an important role as a catalyzer for blocking events, more than influencing substantially their properties once they are realized.

\subsection{Lorenz energy cycle during blocking}

We want to give a physical interpretation to the lower predictability found during blocking events by studying the energetics of CLVs. In a previous work Schubert and Lucarini, 2015, we made use of the fact that the CLVs are covariant solutions of the tangent linear equation and explained the growth and decay rate of the CLVs by looking at their Lorenz energy cycle. We showed that it is possible to study each individual term responsible for energy conversions and sinks derived from the tangent linear equations. For details on that approach we would like to refer to this work. As opposed to the usual analysis of the energetics of linear perturbations of stationary background states, in the case of the CLVs the background is fluctuating and the interactions between the perturbations and the background are more complex. Therefore, let us now briefly summarize how this energy cycle is computed. For each CLV, its average growth rate of the $\left(L^{2}\right)$ square norm coincides with the average growth rate of its energy, thanks to the equivalence of norms in finite dimensional spaces. Hence, we can give a physical interpretation of the changes in the growth rates in the phases where blocking is present versus regular conditions (see section 3.1) by examining the details of the Lorenz energy cycle. We focus here on the budget of the total energy of the $j^{\text {th }} \operatorname{CLV}\left(\psi_{1, j}^{\prime}, \psi_{2, j}^{\prime}\right)$ resulting from the interaction with the background state $\left(\psi_{1}^{B}, \psi_{2}^{B}\right)$ and from dissipative processes. For ease of 


\section{Baroclinic Conversion}
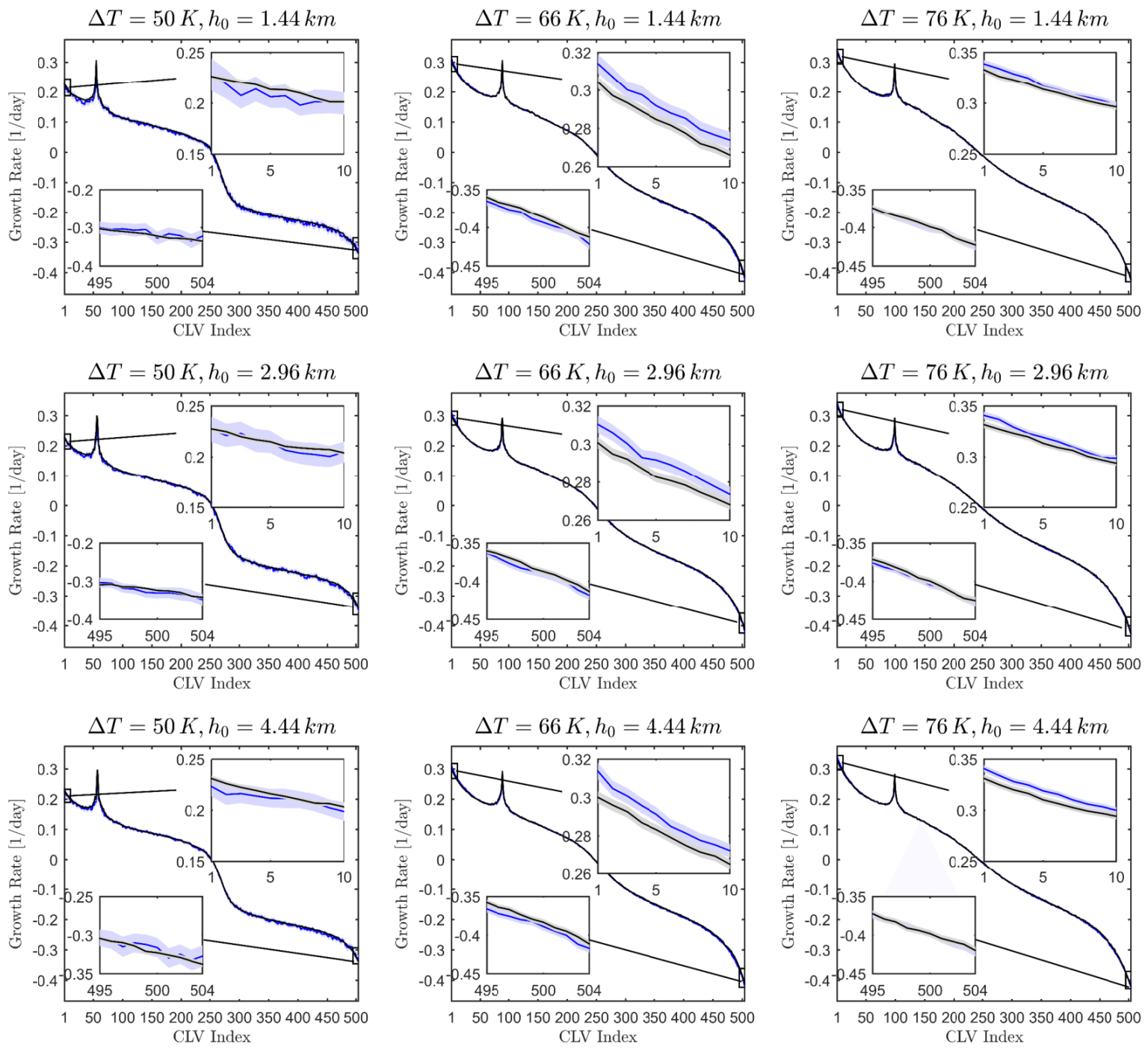

Figure 6: For the nine setups where we observe blocking (see figure 2), the figure compares the baroclinic conversion $\mathcal{C}_{B C}$ during blocking (in blue color) versus unblocked phases (in black color). We additionally show the $3 \sigma$ bars of confidence estimated by computing the degrees of freedom of the time series (shaded areas). For $\Delta T=66 K, 76 K$ we can clearly estimate that for the fastest growing CLVs, the baroclinic conversion increases significantly. For the fastest decaying a negative tendency can be observed, but with weaker statistical significance. For $\Delta T=50 \mathrm{~K}$ such a tendency can not be clearly verified.

notation, we will use for both fields also the barotropic streamfunction $\psi_{P}=\frac{\psi_{1}+\psi_{2}}{2}$ and the baroclinic streamfunction $\psi_{T}=\frac{\psi_{1}-\psi_{2}}{2}$ as well as the respective geostrophic velocities $\mathbf{v}=(u, v)=\left(-\partial_{y} \psi, \partial_{x} \psi\right)$ :

$$
\begin{aligned}
\frac{\mathrm{d}}{\mathrm{dt}} E_{t o t}= & \int d \sigma\left[\Delta \psi_{1, j}^{\prime} \mathbf{v}_{1}^{\prime} \cdot \nabla \psi_{1}^{B}-k_{h}\left(\psi_{1, j}^{\prime} \Delta^{2} \psi_{1, j}^{\prime}\right)\right. \\
& +\langle 1 \leftrightarrow 2\rangle+2 r \psi_{2, j}^{\prime} \Delta \psi_{2, j}^{\prime} \\
& \left.-\frac{2}{S} \psi_{T, j}^{\prime} \mathbf{v}_{P, j}^{\prime} \cdot \nabla \psi_{T}^{B}+2 \frac{\kappa}{S} \psi_{T, j}^{\prime} \Delta \psi_{T, j}^{\prime}-2 \frac{r_{R}}{S} \psi_{T, j}^{\prime 2}\right]
\end{aligned}
$$




\section{Barotropic Conversion}
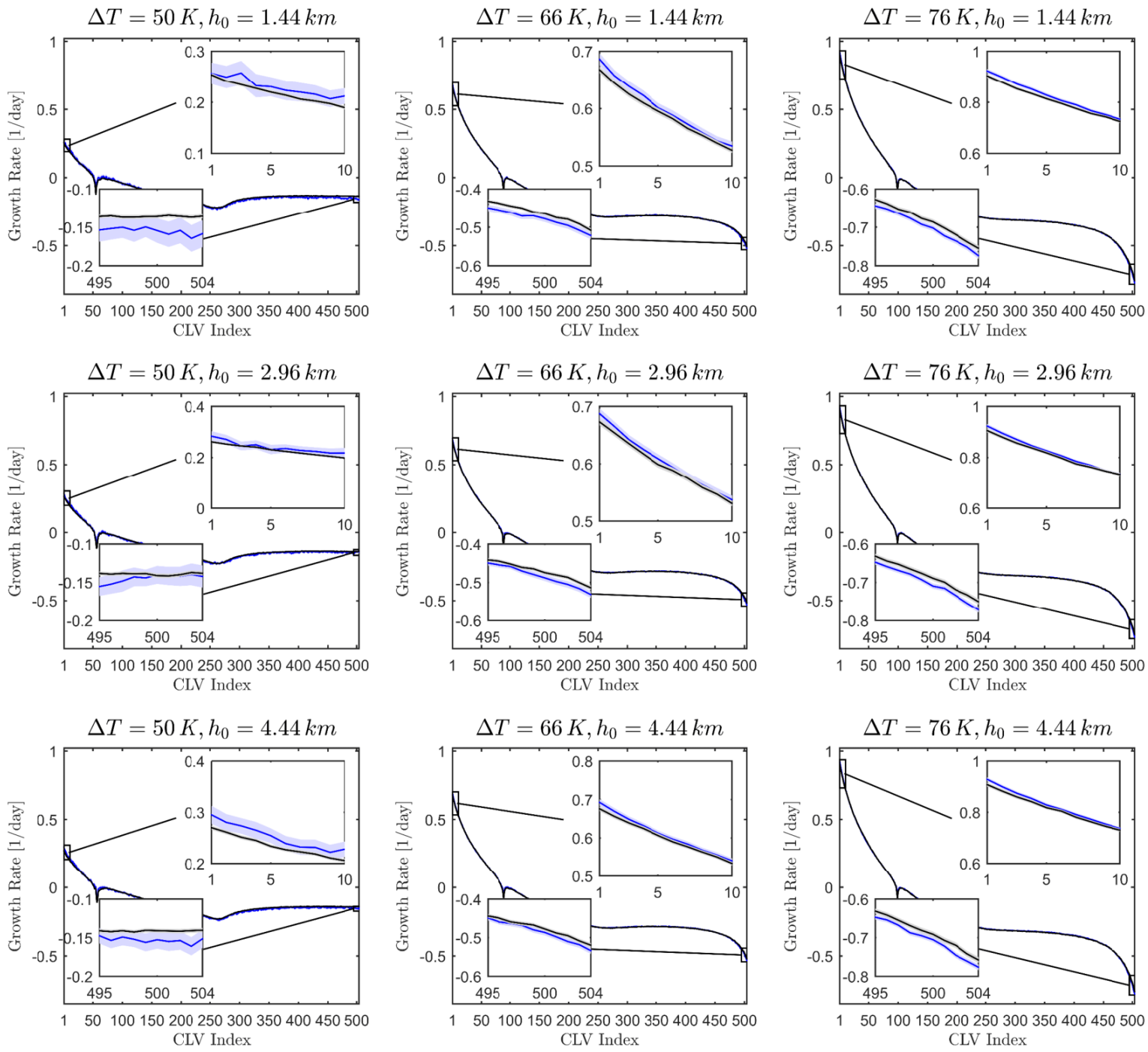

Figure 7: For the nine setups where we observe blocking (see figure 2), the figure shows the differences in the barotropic conversion $\mathcal{C}_{B T}$ during blocking (in blue color) versus unblocked phases (in black color). We additionally show the $3 \sigma$ bars of confidence estimated by computing the degrees of freedom of the time series (shaded areas). For $\Delta T=66 K, 76 K$ we can clearly estimate that for the CLVs with the highest/lowest LEs, the barotropic conversion increases/decreases significantly. For $\Delta T=50 K$ such a tendency can not be clearly verified.

Since the CLVs are growing/decaying perturbations, we will normalize all the following energy conversion terms and sinks by the total energy of the considered CLV. We can group the various terms in equation (9) into the barotropic conversion $\mathcal{C}_{B T}$ which increases to the kinetic energy of the CLVs and the baroclinic conversion $\mathcal{C}_{B C}$ which increases to the potential energy of the CLVs. Hence, both conversions contribute to the total energy of the CLVs. For our investigation, we will sum up all external forcings (newtonian cooling, diffusion and friction) acting on either the potential or kinetic energy in the term S. The energy budget for the total energy is then the following.

$$
\frac{\mathrm{d}}{\mathrm{dt}} E_{t o t}=\mathcal{C}_{B C}+\mathcal{C}_{B T}+S
$$



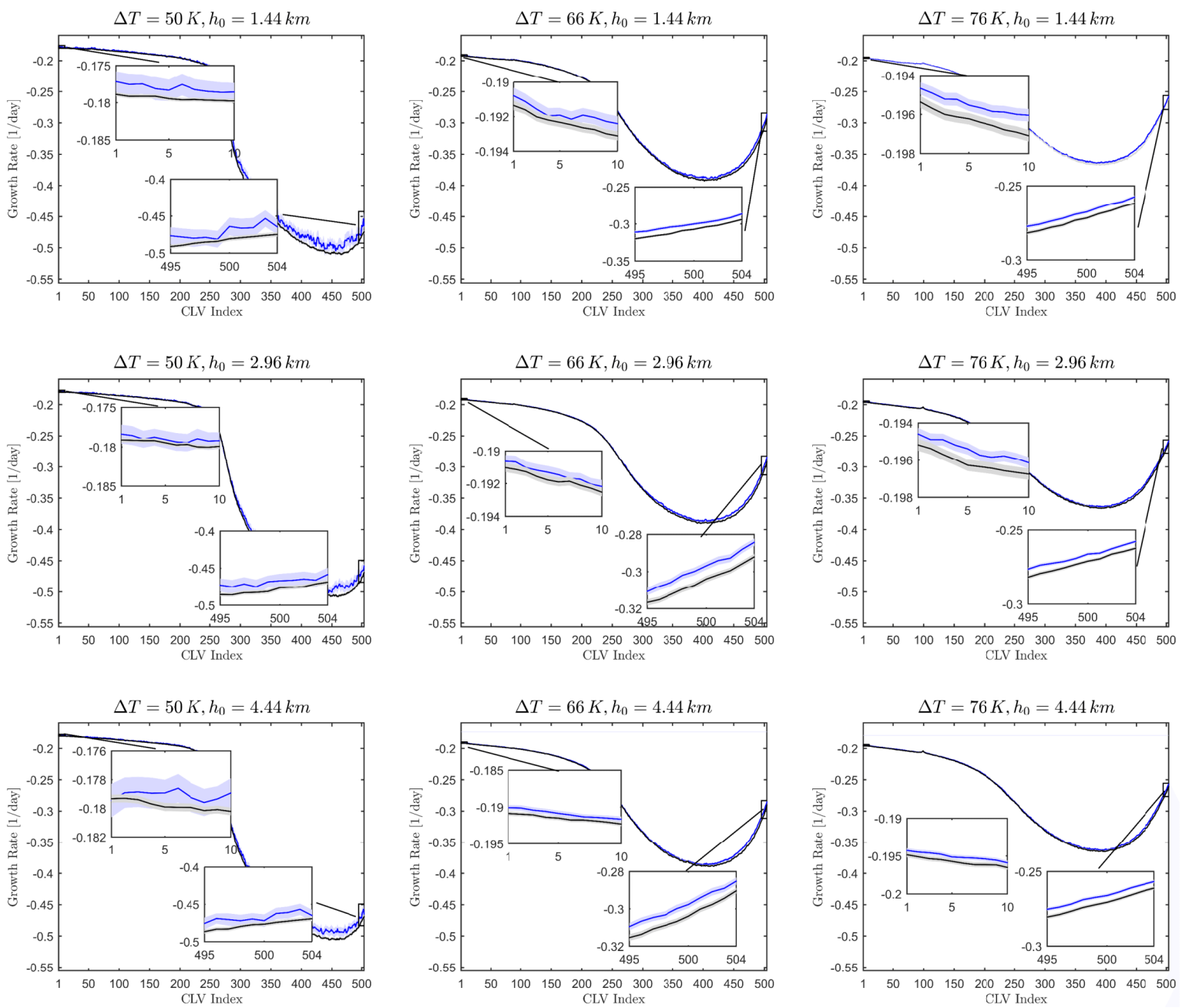

Figure 8: The energy losses of the CLVs are the sum of the Ekman Friction, the heat and eddy diffusivity and newtonian cooling (see [Schubert and Lucarini, 2015]). For the nine setups where we observe blocking (see figure 2), the figure shows the sum of these terms during blocking (in blue color) versus unblocked phases (in black color). We additionally show the $3 \sigma$ bars of confidence estimated by computing the degrees of freedom of the time series (shaded areas). For all $\Delta T$ we can clearly estimate that for the CLVs with the highest/lowest LEs, the energy losses decrease significantly.

The baroclinic and barotropic energy conversion terms are defined as

$$
\mathcal{C}_{B C}=\int d \sigma\left[-\frac{2}{S} \psi_{T, j}^{\prime} \mathbf{v}_{P, j}^{\prime} \cdot \nabla \psi_{T}^{B}\right]
$$

and

$$
\mathcal{C}_{B T}=\int d \sigma\left[\Delta \psi_{1, j}^{\prime} \mathbf{v}_{1, j}^{\prime} \cdot \nabla \psi_{1}^{B}+\Delta \psi_{2, j}^{\prime} \mathbf{v}_{2, j}^{\prime} \cdot \nabla \psi_{2}^{B}\right],
$$


respectively. The energy loss is the sum of newtonian cooling, Ekman friction, and eddy diffusivity.

$$
\begin{aligned}
S= & \int d \sigma\left[-2 k_{h}\left(\psi_{T, j}^{\prime} \Delta^{2} \psi_{T, j}^{\prime}+\psi_{P, j}^{\prime} \Delta^{2} \psi_{P, j}^{\prime}\right)\right. \\
& \left.+r \psi_{2, j}^{\prime} \Delta \psi_{2, j}^{\prime}+2 \frac{\kappa}{S} \psi_{T, j}^{\prime} \Delta \psi_{T, j}^{\prime}-2 \frac{r_{R}}{S} \psi_{T, j}^{\prime 2}\right]
\end{aligned}
$$

A positive average value for the baroclinic term $\mathcal{C}_{\mathcal{B C}}$ implies that available potential energy of the background flow is converted into available potential energy of the $j^{\text {th }}$ CLV. The corresponding thermal fluctuations are then converted into kinetic energy of the CLV. Instead, an average positive value of the barotropic term $\mathcal{C}_{\mathcal{B} \mathcal{T}}$ implies a direct transfer of kinetic energy from the background flow to the $j^{\text {th }}$ CLV. Just like in the standard case of linear perturbations to a stationary background flow, also in this general scenario we have that a positive rate of baroclinic (barotropic) energy conversion rate is associated to a heat (momentum) flux opposite to the temperature (zonal momentum) gradient of the background flow. Such a negative feedback ensures the global stability of the system and is a manifestation of the second law of thermodynamics [Schubert and Lucarini, 2015]. Clearly, it is a necessary condition for the LE corresponding to a CLV to be positive that at least one of the two terms $\mathcal{C}_{\mathcal{B C}}$ or $\mathcal{C}_{\mathcal{B} \mathcal{T}}$ to be positive on the average.

We can now study how the baroclinic and barotropic energy conversion rates are influenced by the presence of blocked flow conditions. The results are shown in figures 6 and 7 for barotropic and baroclinic processes, respectively. For completeness, we have also plotted the result for the energy sinks of the CLVs in figure 8 .

For all analyzed configurations and for all CLVs, blocked conditions support smaller rates of energy dissipation than regular conditions. Nonetheless, such changes are numerically rather small and can be disregarded in the following discussion.

In the case of weak baroclinic forcing $(\Delta T=50 K)$, the difference in the energetics of the CLVs between blocked and normal conditions is borderline or not statistically significant for most CLVs. Despite lack of strong statistical evidence, some useful indications can be given. Looking at the unstable CLV, we observe that during blocked phases the baroclinic conversion is lower than in usual conditions, whereas the opposite holds for the barotropic conversion. Therefore, we have that the (modest) enhanced growth rate of the unstable CLVs observed in blocked conditions (see figure 5) can be attributed to a more efficient barotropic conversion. Looking at the most stable CLVs, the situation is reversed, with barotropic (baroclinic) conversion rates being reduced (increased) in blocked conditions.

The situation changes when considering conditions where stronger baroclinic forcing is imposed on the system $(\Delta T=66 K$ and $\Delta T=76 K)$. We have that blocked conditions are accompanied by stronger baroclinic and stronger barotropic conversion rates for the unstable CLVs, while, conversely, the both conversion rates are reduced when looking at the most stable CLVs.

These results seems to suggest that the energetics of blocking events is fundamentally different in background states featuring weak versus strong Equator to Pole temperature differences. In the former case, blocking is eminently related to modifications to the barotropic instability of the flow, while in the latter case, it results from modifications of both barotropic and baroclinic instabilities. The synergy between the two forms of instability is likely to be responsible for the increase in the number of blocking events for larger values of $\Delta T$. Note that a strong sensitivity of the properties of the low-frequency variability on the intensity of the jet was already envisioned in Benzi et al. 1986 and verified by Ruti et al. [2006.

The properties of the blocked states in terms of the Lorenz energy cycle of the CLVs are weakly dependent on the value of the perturbation orography $h_{0}$, which confirms in physical terms the eminently catalyzing role of orography for blocking.

\subsection{Localization Of CLVs}

We study now the localization of the CLVs during blocking conditions and compare it with what observed in normal unblocked phases. A measure for the localization is given by the temporal variance 
of the CLVs at the grid points on the domain. In the control runs without orography, the variance of the CLVs does not depend on x. Results by Szendro et al. 2008 suggest that if the zonal symmetry is broken due to orography, the CLVs will be localized in the $\mathrm{x}$ direction. In figure 9, we show as an example the variance of three CLVs in the blocked phase and the unblocked phase for the blockings shown previously in figure 3 (for $\Delta T=66 \mathrm{~K}$ and $h_{0}=4.44 \mathrm{~km}$ and blocking at $x_{b}=15859 \mathrm{~km}$ ). The figure shows a clear impact of the blocking on the variance of the CLVs. Overall, the variance is localized in the meridional direction due to the symmetry break associated with the boundary conditions at $y=0, \pi$, but at the location of the blocking the variance is shifted northward. In the zonal direction, the variance has a weak x-dependence (even if we have no reasons to expect zonal symmetry) during the unblocked phase. Away from the blocking, we see again a non zonal disturbance with wave number four in both phases (see section 3.1). Note that at the location of the block in the background state (see figure 3), the CLVs show a minimum of the variance.

Before we discuss the implications of these results, we would like to analyze the variance for all CLVs by slightly reducing the complexity of the data. We average the variance along the meridional direction and focus on the $\mathrm{x}$-dependence only. Furthermore, we do not wish to analyze the average localization of the CLVs, but instead track the variations of the localization. Hence, we compute the ratio of the meridional average of the variance of the streamfunction of the CLVs (upper and lower layer, indicated by the upper indices 1 and 2 , respectively) during blocking at a particular $x_{b}$

$$
\sigma_{x_{b}}^{(1 / 2)}(x)=\frac{1}{\left|T_{x_{b}}\right|} \int_{t \in T_{x_{b}}} d t \int d y\left(\psi_{1 / 2}(x, y, t)^{2}-\left\langle\psi_{1 / 2}\right\rangle^{2}\right)
$$

and during the unblocked phase

$$
\sigma_{\text {unbl }}^{(1 / 2)}(x)=\frac{1}{\left|T_{\text {unbl }}\right|} \int_{t \in T_{\text {unbl }}} d t \int d y\left(\psi_{1 / 2}(x, y, t)^{2}-\left\langle\psi_{1 / 2}\right\rangle^{2}\right) .
$$

Note that $\langle\cdots\rangle$ is the average along the whole available time series of the CLVs. We define the set $T_{x_{b}}$ to contain all time steps where the flow is blocked at $x_{b}$ and $T_{u n b l}$ to contain all time steps which are not blocked. $|T|$ is the length of the respective phases. For this calculation, we transform the spectral fields into a [64x32] grid point field. Hence, we can view the $\sigma$ s as the variance of the grid point amplitudes of the streamfunction during a blocking situation and during non-blocked phases.

We the measure the change in the localization $\Delta \mathcal{L}$ as follows:

$$
\Delta \mathcal{L}^{1 / 2}(x)=\frac{\sigma_{x_{b}}^{1 / 2}(x)}{\sigma_{u n b l}^{1 / 2}(x)}
$$

If $\Delta \mathcal{L}>1$, then a higher activity of the CLV (y-axis) during blocking at a particular zonal coordinate (x-axis) is implied. As an example, figure 10 shows the results of the above equation (14) for the three local maxima of the blocking rate for $\Delta T=66 \mathrm{~K}$ and $h_{0}=4.44 \mathrm{~km}$ (see figure 2). The other setups show similar results. In the figure, the vertical dashed line indicates the position of the peak of orography along the channel. We present results for the the upper layer streamfunction $\psi_{1}$ (left panel) and the lower layer streamfunction $\psi_{2}$ (right panel). We see that the activity of almost all CLVs is higher close to the blocking and lower in the rest of the channel. To be more precise, the CLVs cluster around a region where blocking is detected. Note that the clustering occurs almost regardless of growth rate of the CLVs. Also, the localization in the lower layer is less strong, which explains the reduction in the dissipation during blocked phases discussed before. The variance of the CLVs at the center of the blocking is lower. This indicates that stability is higher in the center of the blocking compared to its borders. In order to clarify unambiguously this point, the adjoint CLVs would have to be considered. These allow for projecting in a meaningful way an arbitrary perturbation onto the non-orthogonal basis given by the CLVs. For a given time horizon, one could then obtain a characteristic growth/decay rate of linear perturbations in an arbitrary region of the flow.

Note that using orthogonal Lyapunov vectors for this analysis would change the results. We can illustrate this by considering the example of optimal perturbations by Buizza and Molteni 1996. 
Here, only the first optimal perturbation localizes close to the blocking, since this vector converges for long optimization times towards the first CLV, the remaining optimal perturbations can not behave in this way (see section 2.2). The results obtained using the physically relevant CLV basis underline that a transition to a blocked state is a change in the flow regime which effects all time scales and processes. Hence, the linear dynamics of blocking events can not be reduced to a small number of changes on certain time scales and consequently, the detection of blocking events should take this into account.

\section{Summary and Conclusions}

The goal of this paper is to study blocking events in a very simplified atmospheric model of the midlatitudes. Blocking events are persistent deviations of the jet stream in the mid-latitudes from the usual quasi zonal symmetry. Naturally, blocked states possess very unusual properties in terms of weather forecast, and it is especially difficult to predicting the onset and decay of blocking events. It is well known that orography plays a major role in fostering the occurrence of blocking. This model is a quasi geostrophic two layer model on a periodic channel with a beta plane approximation and driven by a forced meridional temperature gradient $\Delta T$. In the spirit of previous analysis of blocking, we added an orographic forcing in order to produce enhanced blocking events in the flow Charney and Straus, 1980. As orography, we use a Gaussian bump placed in the middle of the channel. We investigate four different values of $\Delta T(40 \mathrm{~K}, 50 \mathrm{~K}, 66 \mathrm{~K}$ and $76 \mathrm{~K})$ in order to assess different degrees of large scale turbulence. The impact of orography is investigated with three different heights $h_{0}(1.48$ $\mathrm{km}, 2.96 \mathrm{~km}$ and $4.44 \mathrm{~km}$ ).

While such a setting is definitely outdated and insufficient in terms of providing a realistic statistics of blocking events, it provides qualitatively meaningful results and contains some of the essential physical and mathematical ingredients we want to consider, namely the possibility of having a turbulent state featuring a convincing Lorenz energy cycle fuelled by barotropic and baroclinic instabilities and damped by a variety of dissipative effects.

The main plus of such a simple model is that we are able to construct the CLVs, which are the covariant unstable and stable modes of the turbulent flow and provide a physical representation of the natural fluctuations of the flow. CLVs provide a complete descriptions of the dynamics and geometry of the attractor of the system and are useful for providing a new characterization of the properties of blocked vs regular conditions. CLVs are the suitable choice for such an investigation, since they are covariant and independent of a norm. More precisely, they describe a first order approximation nearby trajectories and they evolve following local features of the non-linear background flow. Our objective in this study is to make a first step towards identifying the signature of the blocking events in the CLVs.

The model we consider here is was originally developed in order to analyze the long term averaged properties of CLVs and their capability to explain the variability of the full non linear flow Schubert and Lucarini, 2015]. There, we were solely interested in the long term average of the Lorenz energy cycle of the CLVs and to connect is to their growth or decay rates and property of being unstable or stable, thus linking the physics and the mathematics of the disturbances. The fluctuations of the CLVs or different "weather regimes" in the background state like blocking were not investigated.

In the analysis presented here, we detect the blocking events with an Tibaldi-Molteni scheme Tibaldi and Molteni, 1990. Given the simplicity of the model we use, it is not surprising that

the statistics of the events we label as blocking are only in qualitative agreement with what found in observations for all configurations we consider. Nevertheless, we can show that the detected events are indeed blocking highs which divert the jet stream from its zonal symmetry. In the unblocked phase, it is also comforting to see that, the flow is more zonally symmetric and its mean state exhibits topographic Rossby waves. For higher meridional temperature gradients $\Delta T$, the occurrence of the blocking rates increases, accompanied by a modest increase of its life time. The orography creates localized regions of high blocking rates. Such regions are located downstream of the orographic disturbance and their prominence is more evident when higher mountains are considered. The orographic influence is weaker 
when adopting a stronger baroclinic forcing.

Each CLV is associated to a LE, which measures its average growth (for unstable CLVs) or decay (for stable CLVs) rate. We have analyzed separately the growth rate of the various CLVs during the blocked and regular regimes. Furthermore, the spatial variance during the blocked and unblocked phases is used for the localization of the CLVs. Our results show a significant increase of the growth rate of the leading CLVs during blocked phases. Hence, the flow is more unstable during blocking. This might be interpreted as a trade off effect between increased stability in the blocked regions and less instability elsewhere in the flow. The increased instability indicates also that the regime transition to and from the blocked regime is in general difficult to predict. It should be noted that persistence as we expect it in the case of blocking and stability are not necessarily dependent on each other. We find support for our conclusions in recent results by Faranda et al. 2015 which show that blockings can be connected to an unstable fixpoint in a reduced phase space. In a closely related piece of work, Faranda et al ${ }^{1}$ have shown that the dimensionality of the reduced phase space determining the dynamics of blocking events is higher than corresponding to regular quasi-zonal dynamics by exploiting a connection between extreme value statistics and the local dimension of the dynamical systems underlying attractor. The fact that we not only observe increased instability in the CLVs but also an increased metric entropy during blockings fits very well to these results.

We have complemented the analysis of the instabilities by investigating the Lorenz energy cycle of the various CLVs and looking at the baroclinic and barotropic conversion rates. The enhancement of the growth rate in the blocked phase for the leading unstable CLVs is due to a strengthening of both barotropic and baroclinic conversion rates for intermediate and high values of $\Delta T$. Instead, for low values of $\Delta T$, enhanced instability of the unstable CLVs during blocked phase results from an enhancement of barotropic instability only. This clarifies that the dynamical processes behind blocking events are not the same in conditions of low versus high baroclinicty of the background flow.

The variance of every CLV clusters around the blocked area. This hints for an increased instability at the boundaries of the detected blocking events. Instead, the spatial variance of the unstable CLVs is lower at the center of the blocking, possibly indicating higher local predictability. The analysis via CLVs seems to provide a powerful way to link the occurrence of blocking events to specific modifications in the temporal and spatial properties of unstable processes responsible for energy conversion.

Gallavotti 2014 suggests that in multiscale systems it should be possible to associate the different spatial spatial and temporal scales of motion (e.g. macroscopic, mesoscopic and microscopic) to specific subsets of CLVs and related LEs. In particular, one expects that highly localized (extended) unstable CLVs might be associated to large (small) growth rates resulting from local (global) instabilities.

It is tempting to follow this idea for investigating the complex portfolio of meteorological instabilities, but one needs indeed to consider more complex models than that adopted here. In particular, in a primitive equation model one could see whether it is possible to recognize small scale CLVs with high growth rate associated to mesoscale instabilities, while, additionally, CLVs associated to convective events should be found when non-hydrostatic models are adopted.

Clearly, a QG model like the one used in this study is not appropriate for investigating these interesting aspects. Instead, the fact that here, as already observed in Schubert and Lucarini 2015, all CLVs have similar spatial scale (and the LEs, apart from those very close to zero, are also similar) is a "a posteriori" confirmation of the self-consistency of the scale analysis leading to the QG approximation. Another possibility is, instead, to use the formalism of CLVs and associated LEs to study a fluid model encompassing regions with different inertia and thermal inertia, like in the case of a coupled atmosphere-ocean model, in order to define rigorously, e.g., coupled modes of variability. Vannitsem and Lucarini [2015] have recently provided extremely encouraging results in this direction using a severely truncated coupled atmosphere-ocean model.

Future work should approach the characterization of blockings via CLVs in a model featuring

\footnotetext{
${ }^{1}$ These results are so far unpublished, yet they have been presented at the summer school Statistical and mathematical tools for the study of climate extremes, held in Cargese, France, November 2015 and at the 2015 AGU assembly; see D. Faranda, P. Yiou and M. Carmen Alvarez Castro "Butterflies, Black swans and Dragon kings: How to use the Dynamical Systems Theory to build a "zoology" of mid-latitude circulation atmospheric extremes?" https://agu.confex.com/agu/ fm15/meetingapp.cgi/Paper/72315
} 
spherical geometry and a realistic forcing, e.g. in Vannitsem 2001, in order to assess more persistent blockings. Another direction of work is to define a local stability map of the flow using the adjoint CLVs. These would allow for quantifying the local growth rates of arbitrary linear perturbations to the flow. In this way, it should be possible to unambiguously identify local persistent structures like blocking.

\section{Acknowledgement}

The authors wish to thank O. Talagrand, J. M. Lopez, G. Gallavotti, C. Franzke and F. Lunkeit for helpful discussions on the manuscript. We wish to thank as well T. Frisius for allowing to use and expand his QG model. V.L. acknowledges fundings from the Cluster of Excellence for Integrated Climate Science (CLISAP) and from the European Research Council under the European Community's Seventh Framework Programme (FP7/2007-2013)/ERC Grant agreement No. 257106. S. Schubert acknowledges fundings from the "International Max Planck Research School - Earth System Modeling" (IMPRS - ESM).

\section{References}

Maarten H. P. Ambaum. Unimodality of Wave Amplitude in the Northern Hemisphere. Journal of the Atmospheric Sciences, 65(3):1077-1086, mar 2008. ISSN 0022-4928. doi: 10.1175/2007JAS2298.1. URL http://centaur.reading.ac.uk/846/http://journals.ametsoc.org/doi/abs/10.1175/ 2007JAS2298.1.

F. Baur. Musterbeispiele Europäischer Grosswetterlagen. Dietrich'sche Verlagsbuchhandlung, Wiesbaden, 1947.

R Benzi, P Malguzzi, A Speranza, and A Sutera. The statistical properties of general atmospheric circulation: observational evidence and a minimal theory of bimodality. Quarterly Journal of the Royal Meteorological Society, 1986. ISSN 1477870X.

P. Berrisford, B. J. Hoskins, and E. Tyrlis. Blocking and Rossby Wave Breaking on the Dynamical Tropopause in the Southern Hemisphere. Journal of the Atmospheric Sciences, 64(8):2881-2898, aug 2007. ISSN 0022-4928. doi: 10.1175/JAS3984.1. URL http://journals.ametsoc.org/doi/ abs/10.1175/JAS3984.1.

G. Boffetta, P. Giuliani, G. Paladin, and A. Vulpiani. An Extension of the Lyapunov Analysis for the Predictability Problem. Journal of the Atmospheric Sciences, 55(23):34093416, dec 1998. ISSN 0022-4928. doi: 10.1175/1520-0469(1998)055〈3409:AEOTLA 2.0.CO; 2. URL http://arxiv.org/abs/chao-dyn/9801030http://journals.ametsoc.org/doi/abs/ 10.1175/1520-0469(1998)055<3409:AEOTLA>2.0.C0;2

G. Boffetta, G. Lacorata, S. Musacchio, and A. Vulpiani. Relaxation of finite perturbations: Beyond the fluctuation-response relation. Chaos: An Interdisciplinary Journal of Nonlinear Science, 13(3): 806, 2003. ISSN 10541500. doi: 10.1063/1.1579643. URL http://scitation. aip.org/content/ aip/journal/chaos/13/3/10.1063/1.1579643.

Roberto Buizza and Franco Molteni. The Role of Finite-Time Barotropic Instability during Transition to Blocking. Journal of the Atmospheric Sciences, 53(12):1675-1697, 1996. ISSN 0022-4928. doi: 10.1175/1520-0469(1996)053〈1675:TROFTB $\rangle 2.0$. CO;2.

J. G. Charney and J. G. DeVore. Multiple Flow Equilibria in the Atmosphere and Blocking. Journal of the Atmospheric Sciences, 36(7):1205-1216, jul 1979. ISSN 0022-4928. doi: 10.1175/1520-0469(1979)036〈1205:MFEITA $\rangle$ 2.0.CO;2. URL http://journals . amet soc .org/doi/ abs/10.1175/1520-0469(1979)036<1205:MFEITA>2.0.C0;2. 
Jule G. Charney and David M. Straus. Form-Drag Instability, Multiple Equilibria and Propagating Planetary Waves in Baroclinic, Orographically Forced, Planetary Wave Systems. Journal of the Atmospheric Sciences, 37(6):1157-1176, jun 1980. ISSN 0022-4928. doi: 10.1175/ 1520-0469(1980)037〈1157:FDIMEA $\rangle$ 2.0.CO;2. URL http://journals . ametsoc.org/doi/abs/10. 1175/1520-0469(1980)037<1157:FDIMEA>2.0.CD;2.

D. T. Crommelin. Regime Transitions and Heteroclinic Connections in a Barotropic Atmosphere. Journal of the Atmospheric Sciences, 60(2):229-246, 2003. ISSN 0022-4928. doi: 10.1175/ 1520-0469(2003)060<0229:RTAHCI $>2.0 . \mathrm{CO} ; 2$.

J. Eckmann and D. Ruelle. Ergodic theory of chaos and strange attractors. Reviews of Modern Physics, 57(3):617-656, jul 1985. ISSN 0034-6861. doi: 10.1103/RevModPhys.57.617. URL http: //link.aps.org/doi/10.1103/RevModPhys.57.617.

Davide Faranda, Giacomo Masato, Nicholas Moloney, Yuzuru Sato, Francois Daviaud, Bérengère Dubrulle, and Pascal Yiou. The switching between zonal and blocked mid-latitude atmospheric circulation: a dynamical system perspective. Climate Dynamics, dec 2015. ISSN 0930-7575. doi: 10.1007/s00382-015-2921-6. URL https://hal .archives-ouvertes.fr/hal-01136648http: //link.springer.com/10.1007/s00382-015-2921-6.

G Floquet. Sur les equations differentielles lineaires. Ann. ENS [2], 12:47-88, 1883. URL http://archive.numdam.org/ARCHIVE/ASENS/ASENS \{_\}1884\{_\}3\{_\}1\{_\}/ ASENS $\left\{_{-}\right\} 1884\left\{_{-}\right\} 3\left\{_{-}\right\} 1\left\{_{-}\right\}\left\{_{-}\right\} 181\left\{_{-}\right\} 0 /$ ASENS $\left._{-}\right\} 1884\left\{_{-}\right\} 3\left\{_{-}\right\} 1\left\{_{-}\right\}\left\{_{-}\right\} 181\left\{_{-}\right\} 0 . p d f$

JS Frederiksen. Adjoint Sensitivity and Finite-Time Normal Mode Disturbances during Blocking. Journal of the atmospheric sciences, 54(1989):1144-1165, 1997. URL http://journals ametsoc . org/doi/abs/10.1175/1520-0469(1997)054<1144:ASAFTN>2.0.C0;2.

Gary Froyland, Thorsten Hüls, Gary P. Morriss, and Thomas M. Watson. Computing covariant Lyapunov vectors, Oseledets vectors, and dichotomy projectors: A comparative numerical study. Physica D: Nonlinear Phenomena, 247(1):18-39, mar 2013. ISSN 01672789. doi: 10.1016/j.physd. 2012.12.005. URL http://linkinghub.elsevier.com/retrieve/pii/S0167278912003090.

G. Gallavotti and E. G D Cohen. Dynamical ensembles in stationary states. Journal of Statistical Physics, 80(5-6):931-970, sep 1995. ISSN 0022-4715. doi: 10.1007/BF02179860. URL http: //link.springer.com/10.1007/BF02179860.

Giovanni Gallavotti. Nonequilibrium and Irreversibility. Theoretical and Mathematical Physics. Springer International Publishing, Cham, 2014. ISBN 978-3-319-06757-5. doi: 10.1007/ 978-3-319-06758-2. URL http://arxiv.org/abs/1311.6448http://link.springer.com/10. 1007/978-3-319-06758-2.

F. Ginelli, P. Poggi, A. Turchi, H. Chaté, R. Livi, and A. Politi. Characterizing dynamics with covariant Lyapunov vectors. Physical Review Letters, 99(13):130601, sep 2007. ISSN 0031-9007. doi: 10.1103/PhysRevLett.99.130601. URL http://arxiv.org/abs/0706.0510.

A Gritsun. Statistical characteristics, circulation regimes and unstable periodic orbits of a barotropic atmospheric model. Philosophical Transactions of the Royal Society of London A: Mathematical, Physical and Engineering Sciences, 371(1991), apr 2013. URL http://rsta. royalsocietypublishing.org/content/371/1991/20120336.abstract.

James R. Holton. An Introduction to Dynamic Meteorology, Volume 1. Academic Press, 2004. ISBN 9780123848666. URL http://books.google.com/books?id=fhW5oDv3EPsC\{\&\}pgis=1

D. Kondrashov, K. Ide, and M. Ghil. Weather Regimes and Preferred Transition Paths in a ThreeLevel Quasigeostrophic Model. Journal of the Atmospheric Sciences, 61(5):568-587, 2004. ISSN 0022-4928. doi: 10.1175/1520-0469(2004)061〈0568:WRAPTP $\rangle 2.0 . \mathrm{CO} ; 2$. 
P. V. Kuptsov and U. Parlitz. Theory and Computation of Covariant Lyapunov Vectors. Journal of Nonlinear Science, 22(5):727-762, mar 2012. ISSN 0938-8974. doi: 10.1007/s00332-012-9126-5. URL http://link.springer.com/10.1007/s00332-012-9126-5.

B Legras and R Vautard. A Guide to Liapunov vectors. In Proceedings 1995 ECMWF Seminar on Predictability, volume 1, pages 143-156, 1996.

C. E. Leith. The standard error of time-average estimates of climatic means. Journal of Applied Meteorology, 12(6):1066 - 1069, 1973. doi: 10.1175/1520-0450(1973)012\{\\%\}253C1066\{\\% \}253ATSEOTA $\{\backslash \%\} 253 \mathrm{E} 2.0 . \mathrm{CO}\{\backslash \%\} 253 \mathrm{~B} 2$. URL http://journals.ametsoc.org/doi/abs/10. 1175/1520-0450(1973) 012<1066:TSEOTA >2.0.CO;2.

H. Lejenäs and H. $\{\backslash \mathrm{O}\}$ kland. Characteristics of northern hemisphere blocking as determined from a long time series of observational data, 1983. ISSN 0280-6495.

E. N. Lorenz. Deterministic Nonperiodic Flow. Journal of the Atmospheric Sciences, 20(2):130141, mar 1963. ISSN 0022-4928. doi: 10.1175/1520-0469(1963)020<0130:DNF $\rangle 2.0 . C O ; 2$. URL http://journals.ametsoc.org/doi/abs/10.1175/1520-0469(1963)020<0130:DNF>2.0.C0;2.

V. Lucarini and S. Sarno. A statistical mechanical approach for the computation of the climatic response to general forcings. Nonlinear Processes in Geophysics, 18(1):7-28, 2011. ISSN 10235809. doi: $10.5194 /$ npg-18-7-2011.

V. Lucarini, A. Speranza, and R. Vitolo. Parametric smoothness and self-scaling of the statistical properties of a minimal climate model: What beyond the mean field theories? Physica D: Nonlinear Phenomena, 234(2):105-123, oct 2007. ISSN 01672789. doi: 10.1016/j.physd.2007.07.006. URL http://linkinghub.elsevier.com/retrieve/pii/S0167278907002345.

Valerio Lucarini, Richard Blender, Corentin Herbert, Francesco Ragone, Salvatore Pascale, and Jeroen Wouters. Mathematical and physical ideas for climate science. Reviews of Geophysics, 52(4):809 859, 2014. ISSN 87551209. doi: 10.1002/2013RG000446. URL http://arxiv.org/abs/1311. 1190http://doi.wiley.com/10.1002/2013RG000446.

Franco Molteni, Alfonso Sutera, and Nicoletta Tronci. The EOFs of the Geopotential Eddies at 500 mb in Winter and Their Probability Density Distributions, 1988. ISSN 0022-4928.

Manfred Mudelsee. Climate Time Series Analysis, volume 42 of Atmospheric and Oceanographic Sciences Library. Springer Netherlands, Dordrecht, 2010. ISBN 978-90-481-9481-0. doi: 10.1007/ 978-90-481-9482-7. URL http://link.springer.com/10.1007/978-90-481-9482-7.

Hiroaki Naoe and Yoshihisa Matsuda. Rossby Wave Propagation and Blocking Formation in Realistic Basic Flows. Journal of the Meteorological Society of Japan, 80(4):717-731, 2002. ISSN 0026-1165. doi: $10.2151 / \mathrm{jmsj} .80 .717$.

Gregor Nitsche, John M. Wallace, and Charles Kooperberg. Is There Evidence of Multiple Equilibria in Planetary Wave Amplitude Statistics? Journal of the Atmospheric Sciences, 51(2):314-322, 1994. ISSN 0022-4928. doi: 10.1175/1520-0469(1994)051〈0314:ITEOME〉2.0.CO;2.

Diego Pazó, Miguel a. Rodríguez, and Juan M. López. Spatio-temporal evolution of perturbations in ensembles initialized by bred, Lyapunov and singular vectors. Tellus, Series A: Dynamic Meteorology and Oceanography, 62(1):10-23, 2010. ISSN 02806495. doi: 10.1111/j.1600-0870.2009.00419.x.

J. L. Pelly and B. J. Hoskins. A New Perspective on Blocking. Journal of the Atmospheric Sciences, 60(5):743-755, mar 2003. ISSN 0022-4928. doi: 10.1175/1520-0469(2003)060〈0743:ANPOB $\rangle$ 2. 0.CO;2. URL http://centaur.reading.ac.uk/5285/http://journals.ametsoc.org/doi/abs/ 10.1175/1520-0469(2003)060<0743:ANPOB >2.0.CO;2. 
N. A. Phillips. The general circulation of the atmosphere: A numerical experiment. Quarterly Journal of the Royal Meteorological Society, 82(352):123-164, apr 1956. ISSN 00359009. doi: 10.1002/qj. 49708235202. URL http://doi.wiley.com/10.1002/qj .49708235202.

Brian B. Reinhold and Raymond T. Pierrehumbert. Dynamics of Weather Regimes: Quasi-Stationary Waves and Blocking. Monthly Weather Review, 110(9):1105-1145, sep 1982. ISSN 0027-0644. doi: 10.1175/1520-0493(1982)110〈1105:DOWRQS $\rangle 2.0 . C O ; 2 . \quad$ URL http://journals.ametsoc. org/doi/abs/10.1175/1520-0493(1982) 110<1105:DOWRQS>2.0.C0;2.

D. F. Rex. Blocking Action in the Middle Troposphere and its Effect upon Regional Climate. Tellus A, 2(3):196-211, aug 1950. ISSN 0280-6495. doi: 10.3402/tellusa.v2i4.8603. URL http://tellusa. net/index.php/tellusa/article/view/8546.

D. Ruelle. Ergodic theory of differentiable dynamical systems. Publications mathématiques de l'IHÉS, 50(1):27-58, dec 1979. ISSN 0073-8301. doi: 10.1007/BF02684768. URL http://link. springer. com/article/10.1007/BF02684768http://link.springer.com/10.1007/BF02684768

David Ruelle. A review of linear response theory for general differentiable dynamical systems. Nonlinearity, 22(4):855-870, apr 2009. ISSN 0951-7715. doi: 10.1088/ 0951-7715/22/4/009. URL http://stacks.iop.org/0951-7715/22/i=4/a=009?key=crossref . cd1887e2608dc1739e5a5efada7981e1.

Paolo M. Ruti, Valerio Lucarini, Alessandro Dell'Aquila, Sandro Calmanti, and Antonio Speranza. Does the subtropical jet catalyze the midlatitude atmospheric regimes? Geophysical Research Letters, 33(6):L06814, 2006. ISSN 0094-8276. doi: 10.1029/2005GL024620. URL http://doi. wiley.com/10.1029/2005GL024620

R. M. Samelson. Lyapunov, Floquet, and singular vectors for baroclinic waves. Nonlinear Processes in Geophysics, 8(6):439-448, 2001. ISSN 1607-7946. doi: 10.5194/npg-8-439-2001. URL http://www.nonlin-processes-geophys.net/8/439/2001/npg-8-439-2001.pdfhttp:// www.nonlin-processes-geophys.net/8/439/2001/.

Bernd Schalge, Richard Blender, and Klaus Fraedrich. Blocking Detection Based on Synoptic Filters. Advances in Meteorology, 2011(1):1-11, 2011. ISSN 1687-9309. doi: 10.1155/2011/717812.

Sebastian Schubert and Valerio Lucarini. Covariant Lyapunov vectors of a quasi-geostrophic baroclinic model: analysis of instabilities and feedbacks. Quarterly Journal of the Royal Meteorological Society, pages n/a-n/a, jul 2015. ISSN 00359009. doi: 10.1002/qj.2588. URL http://doi.wiley.com/10. $1002 / q j .2588$

Mario Sempf, Klaus Dethloff, Dörthe Handorf, and Michael V. Kurgansky. Toward Understanding the Dynamical Origin of Atmospheric Regime Behavior in a Baroclinic Model, 2007. ISSN 0022-4928.

A. Speranza and P. Malguzzi. The Statistical Properties of a Zonal Jet in a Baroclinic Atmosphere: A Semilinear Approach. Part I: Quasi-geostrophic, Two-Layer Model Atmosphere. Journal of the Atmospheric Sciences, 45(21):3046-3062, nov 1988. ISSN 0022-4928. doi: 10.1175/ 1520-0469(1988)045/3046:TSPOAZ〉2.0.CO;2. URL http://journals . ametsoc.org/doi/abs/10. 1175/1520-0469(1988) 045<3046:TSPOAZ>2.0.CO;2.

Alfonso Sutera. Probability density distribution of large-scale atmospheric flow. Advances in Geophysics, 29:227-249, 1986.

Ivan G. Szendro, Juan M. López, and Miguel a. Rodríguez. Dynamics of perturbations in disordered chaotic systems. Physical Review E - Statistical, Nonlinear, and Soft Matter Physics, 78(3):1-10, 2008. ISSN 15393755. doi: 10.1103/PhysRevE.78.036202. 
Alexis Tantet, Fiona R. van der Burgt, and Henk a. Dijkstra. An early warning indicator for atmospheric blocking events using transfer operators. Chaos: An Interdisciplinary Journal of Nonlinear Science, 25(3):036406, 2015. ISSN 1054-1500. doi: 10.1063/1.4908174. URL http: //scitation.aip.org/content/aip/journal/chaos/25/3/10.1063/1.4908174.

S. Tibaldi and F. Molteni. On the operational predictability of blocking. Tellus A, 42(3):343-365, may 1990. ISSN 0280-6495. doi: 10.1034/j.1600-0870.1990.t01-2-00003. x. URL http://journals.sfu.ca/coaction/index.php/tellusa/article/view/11882http: //tellusa.net/index.php/tellusa/article/view/11882.

A. Trevisan and F. Pancotti. Periodic Orbits, Lyapunov Vectors, and Singular Vectors in the Lorenz System. Journal of the Atmospheric Sciences, 55(3):390-398, feb 1998. ISSN 0022-4928. doi: 10.1175/1520-0469(1998)055〈0390:POLVAS $\rangle$ 2.0.CO;2. URL http://journals . ametsoc.org/doi/ $\mathrm{abs} / 10.1175 / 1520-0469(1998) 055<0390$ : POLVAS>2.0.CO;2.

K. K. Tung and A. J. Rosenthal. Theories of Multiple Equilibria-A Critical Reexamination. Part I: Barotropic Models. Journal of the Atmospheric Sciences, 42(24):2804-2819, dec 1985. ISSN 0022-4928. doi: 10.1175/1520-0469(1985)042/2804:TOMEAC $\rangle 2.0 . C O ; 2$. URL http://journals . ametsoc.org/doi/abs/10.1175/1520-0469(1985)042<2804:TOMEAC>2.0.CO;2

S. Vannitsem. Toward a phase-space cartography of the short and medium-range predictability of weather regimes. Tellus, Series A: Dynamic Meteorology and Oceanography, 53(1):56-73, 2001. ISSN 02806495. doi: 10.3402/tellusa.v53i1.12180.

Stephane Vannitsem and Valerio Lucarini. Statistical and Dynamical Properties of Covariant Lyapunov Vectors in a Coupled Atmosphere-Ocean Model-Multiscale Effects, Geometric Degeneracy, and Error Dynamics. arXiv preprint arXiv:1510.00298, 2015.

C. L. Wolfe and R. M. Samelson. Normal-Mode Analysis of a Baroclinic Wave-Mean Oscillation. Journal of the Atmospheric Sciences, 63(11):2795-2812, nov 2006. ISSN 0022-4928. doi: 10.1175/ JAS3788.1. URL http://journals.ametsoc.org/doi/abs/10.1175/JAS3788.1.

C. L. Wolfe and R. M. Samelson. An efficient method for recovering Lyapunov vectors from singular vectors. Tellus A, 59(3):355-366, may 2007. ISSN 0280-6495. doi: 10.1111/j.1600-0870.2007.00234.x. URL http://tellusa.net/index.php/tellusa/article/view/15003.

Christopher L. Wolfe and Roger M. Samelson. Singular Vectors and Time-Dependent Normal Modes of a Baroclinic Wave-Mean Oscillation. Journal of the Atmospheric Sciences, 65(3):875-894, 2008. ISSN 0022-4928. doi: 10.1175/2007JAS2364.1. 
$x_{b} \approx 15859 \mathrm{~km}$ for $\Delta T=66 \mathrm{~K}$ and $h_{0}=4.44 \mathrm{~km}$

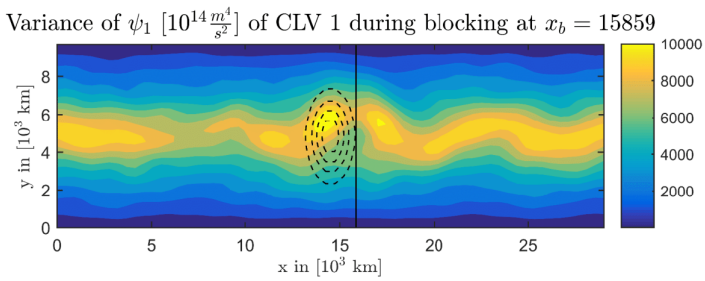

Variance of $\psi_{2}\left[10^{14} \frac{m^{4}}{s^{2}}\right]$ of CLV 1 during blocking at $x_{b}=15859 \mathrm{~km}$
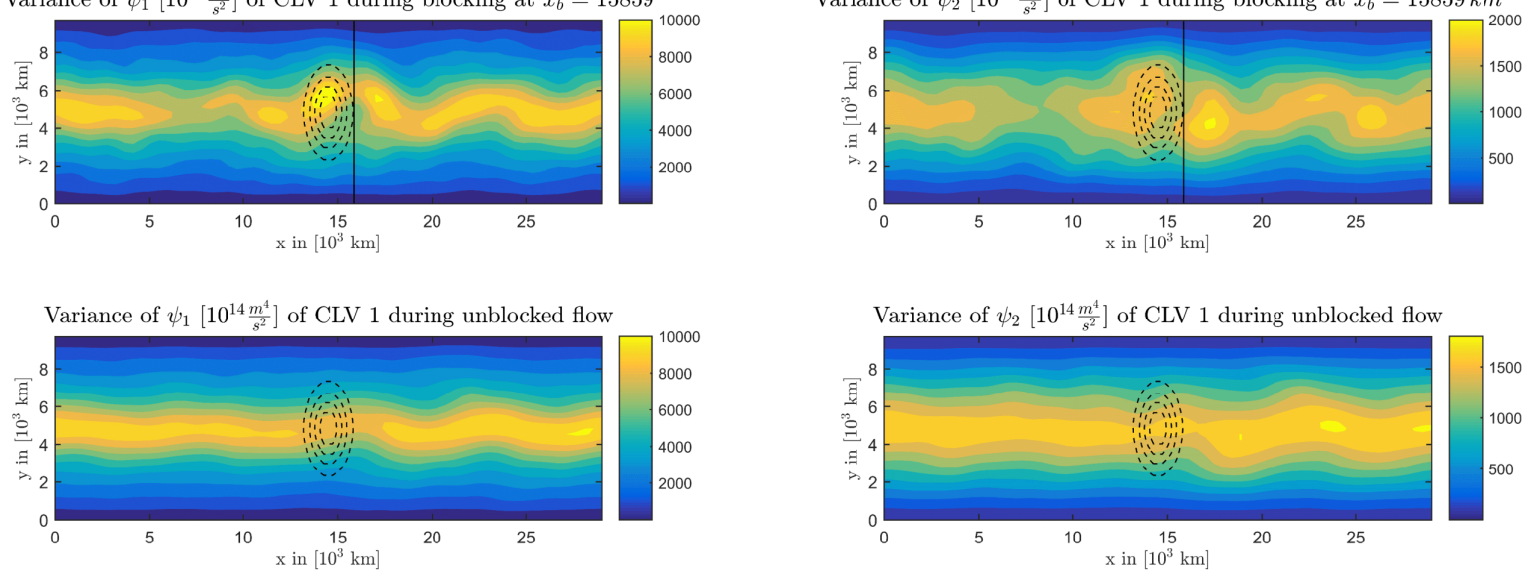

(a) The first CLV

$x_{b} \approx 15859 \mathrm{~km}$ for $\Delta T=66 K$ and $h_{0}=4.44 \mathrm{~km}$

Variance of $\psi_{1}\left[10^{14} \frac{m^{4}}{s^{2}}\right]$ of CLV 10 during blocking at $x_{b}=15859$
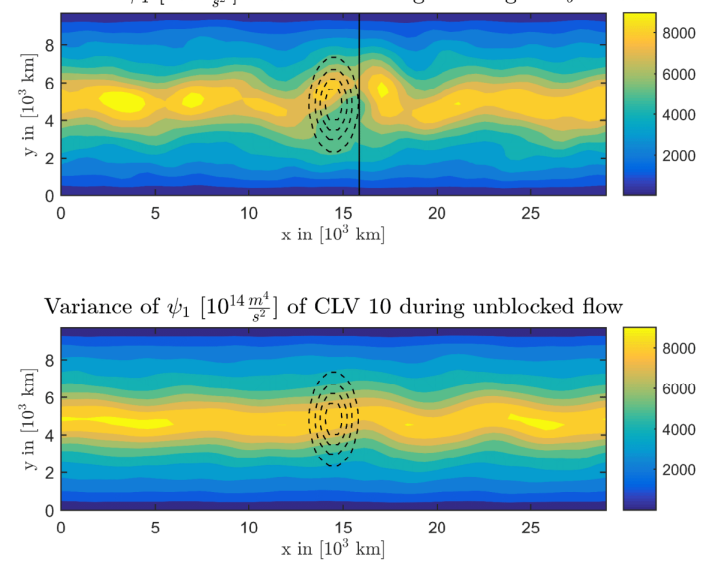

Variance of $\psi_{2}\left[10^{14} \frac{m^{4}}{s^{2}}\right]$ of CLV 10 during blocking at $x_{b}=15859 \mathrm{~km}$

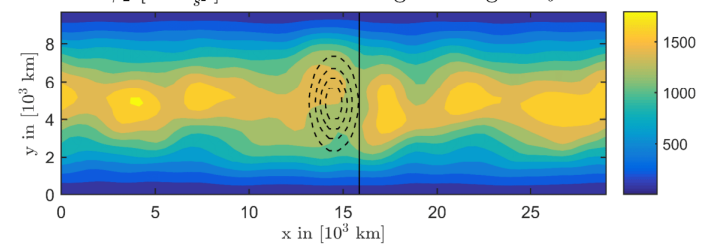

Variance of $\psi_{2}\left[10^{14} \frac{m^{4}}{s^{2}}\right]$ of CLV 10 during unblocked flow

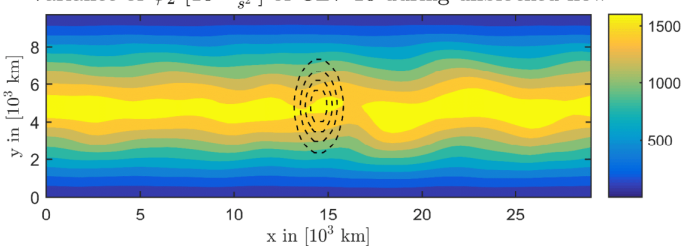

(b) The $10^{\text {th }} \mathrm{CLV}$

$x_{b} \approx 15859 \mathrm{~km}$ for $\Delta T=66 \mathrm{~K}$ and $h_{0}=4.44 \mathrm{~km}$

Variance of $\psi_{1}\left[10^{14} \frac{m^{4}}{s^{2}}\right]$ of CLV 100 during blocking at $x_{b}=15859$
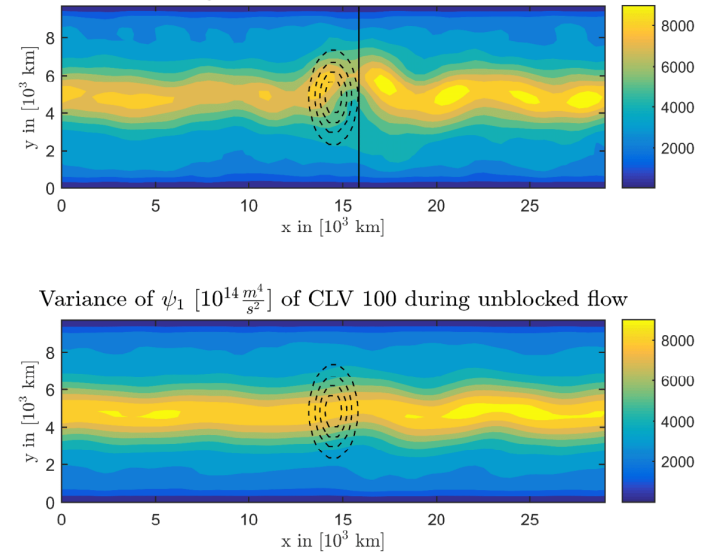

Variance of $\psi_{2}\left[10^{14} \frac{m^{4}}{\mathrm{~s}^{2}}\right]$ of CLV 100 during blocking at $x_{b}=15859 \mathrm{~km}$
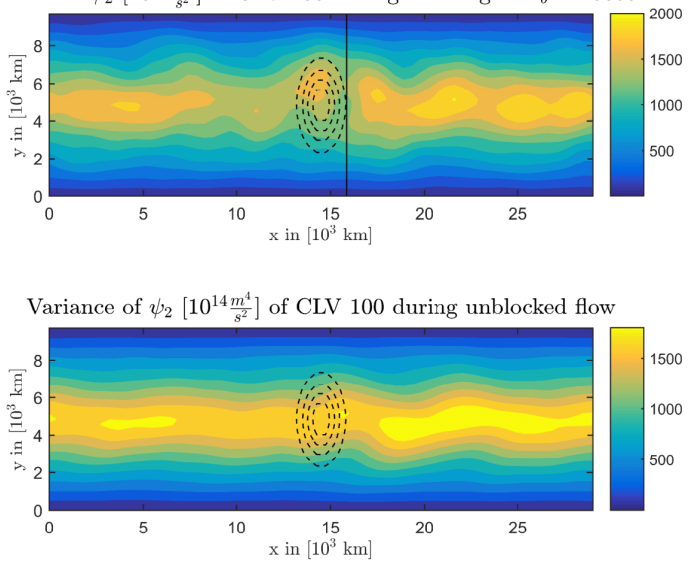

(c) The $100^{\text {th }}$ CLV

Figure 9: Variance of selected covariant Lyapunov vectors. Panel (a) shows the first CLV, (b) the $10^{t h}$ and Panel (c) shows the $100^{t h}$ Panel. The left side of the Panels shows the upper layer stream function $\psi_{1}$ an d the right side shows the upper layer stream function $\psi_{2}$. The upper panels show the average for blocking at $x_{b}=15859 \mathrm{~km}$. The lower panels show the average over the unblocked phase. The vertical black line shows the location of the 3 J locked coordinate, whereas the dashed lines show the location of orography. 
Ratio of variance during blocking at one Gridpoint and variance in the unblocked state

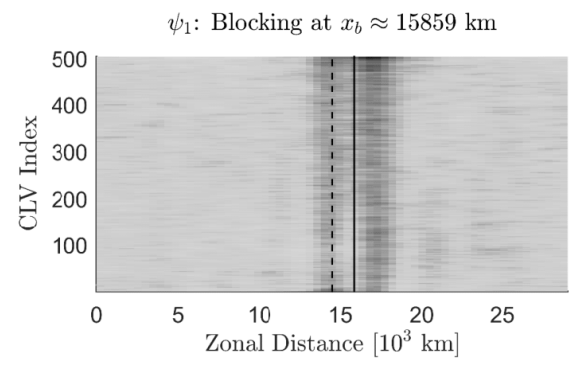

$\psi_{1}$ : Blocking at $x_{b} \approx 22656 \mathrm{~km}$

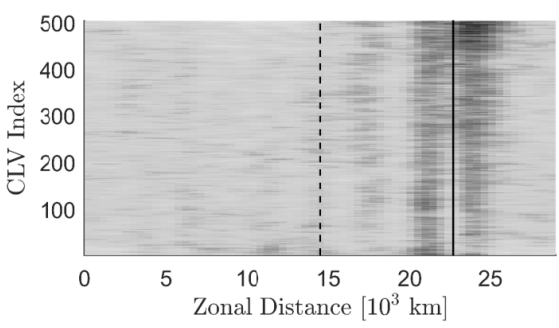

$\psi_{1}$ : Blocking at $x_{b} \approx 906 \mathrm{~km}$

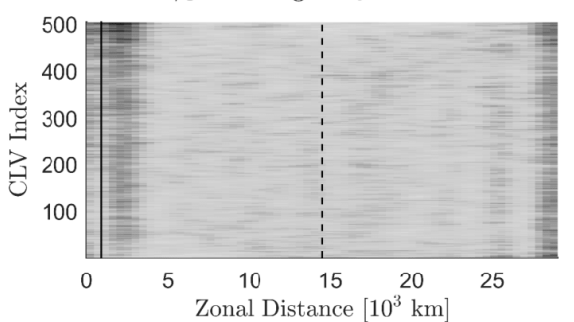

$\Delta T=66 \mathrm{~K}, \mathrm{~h}=2.96 \mathrm{~km}$
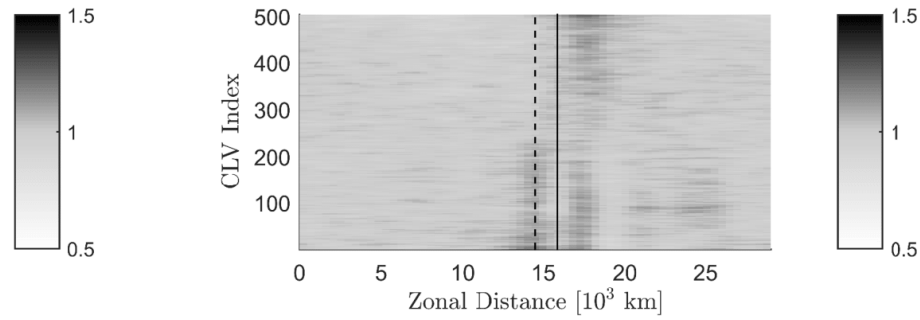

$\psi_{2}$ : Blocking at $x_{b} \approx 22656 \mathrm{~km}$
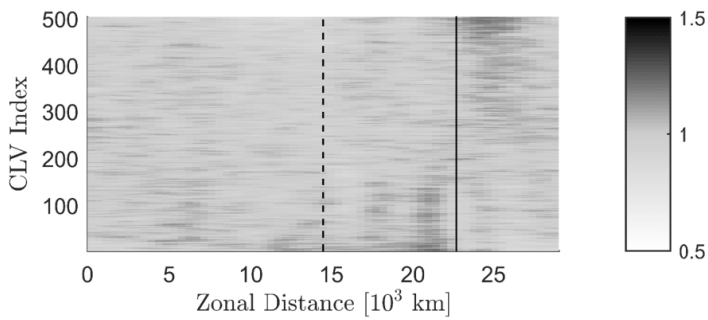

$\psi_{2}$ : Blocking at $x_{b} \approx 906 \mathrm{~km}$
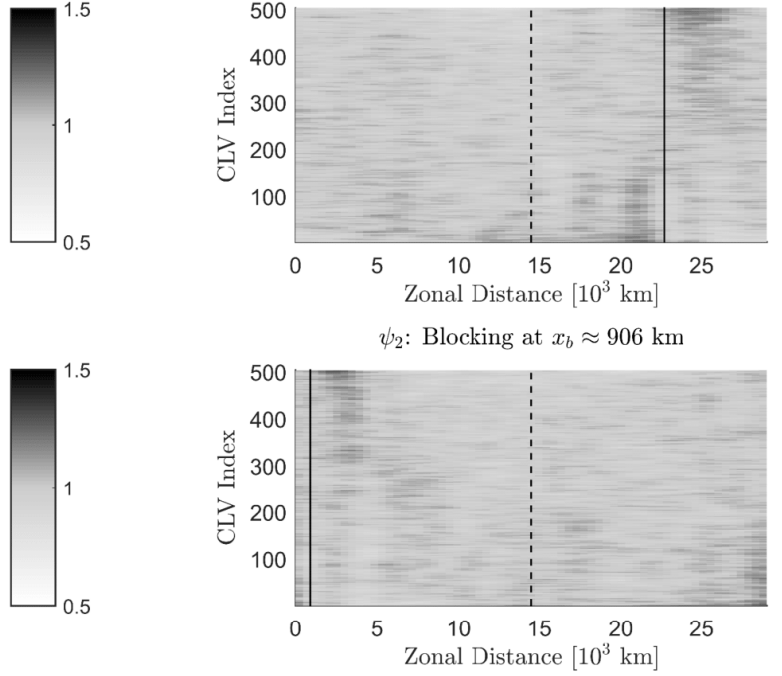

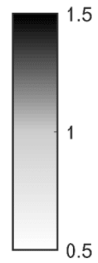

Figure 10: Since the localization of the CLVs is similar for the different setups with blocking events, we show here only results for $\Delta T=66 K$ and $h=4.44 \mathrm{~km}$. For every CLV (y-axis) we show the quotient of the meridionally averaged variance during blocking at a particular $\mathrm{x}$ coordinate (vertical solid black lines) and unblocked phases $(\Delta \mathcal{L}$ in equation $(14)$ ). 